\title{
Configurations du sujet subalterne chez Abla Farhoud Du mutisme féminin aux enjeux de la vocalité
}

\author{
Cécilia W. Francis \\ St. Thomas University
}

Dans son étude consacrée à la démystification des procédés discriminatoires d'ordre impérialiste et patriarcal régissant la socialisation de femmes dites subalternes, Gayatri Chakravorty Spivak a mis en lumière la rature représentative de l'archive dont elles sont victimes et les défis associés à la saisie de leur « voix-conscience » $(2009, \text { p. 49 })^{1}$. De manière parallèle, Hélène Cixous s'est intéressée à la question de l'aphonie féminine, qu'elle a examinée en tant que phénomène d'exclusion résultant

\footnotetext{
${ }^{1}$ Il s'agit de son étude magistrale intitulée, «Can the Subaltern Speak? », datant de 1988, dont la traduction française publiée sous forme d'ouvrage, Les subalternes peuvent-elles parler?, paraît en 2009. Tous les renvois à l'étude en question se feront à partir de cette édition française.
} 
d'une épistémologie philosophique basée sur la suprématie du logos (1976, p. 8). Ces théoriciennes ont circonscrit divers obstacles auxquels sont confrontés certains sujets féminins dans leur accession à une figuration et à un encodage vocal au niveau de la doxa et ont proposé, chacune selon des approches distinctes, des moyens pour retracer leurs effets de présence et leurs échos. Construisant des univers fictifs permettant d'explorer la question cruciale de l'expression vocale chez des héroïnes traditionnellement effacées, Abla Farhoud recoupe et éclaire bon nombre des postulats élaborés par Spivak et par Cixous. Elle s'intéresse aux facteurs conduisant au mutisme de ses protagonistes féminins et forge des outils énonciatifs et langagiers pour rendre leurs interventions idiolectales audibles. Participant d'une modélisation fluctuante mais relativement invariable, ce genre de combat définit le sort de Dounia, une immigrante libanaise illettrée, condamnée au silence sa vie durant, dans Le bonheur a la queue glissante, et la narratrice de Splendide Solitude, enlisée dans des schèmes de soumission féminine rappelant une aliénation coloniale. Chez chaque protagoniste féminin, l'émergence d'une voix singulière s'avère un procès traversé de tensions en raison de phénomènes sociopsychiques de subordination et d'interdiction qui les empêchent de s'épanouir en tant que sujet agissant de la verbalisation.

L'objectif de la présente étude consiste à analyser le cas de ces deux protagonistes farhoudiens au prisme de la notion centrale de sujet subalterne afin d'éclairer les circonstances dictant le mutisme féminin et de cibler les modalités énonciatives et rhétoriques qu'elles adoptent pour le briser. Quoique la critique se soit intéressée aux questions liées au statut du personnage féminin dans l'œuvre théâtrale et 
romanesque de Farhoud, elle a eu tendance à privilégier l'optique de la migrance dans ses argumentations (Oore, 2000; Proulx, 2004; Montandon, 2006; Marcheix, 2010). Or, dans l'un de ses rares textes où elle réfléchit aux problématiques qui la préoccupent, Farhoud manifeste le souci d'ouvrir le thème de l'immigration sur une vision plus universelle du monde. Parlant de son texte dramatique, Les Filles du 5-10-15ф, elle précise : « je n'écrivais pas une pièce sur deux jeunes immigrantes, mais surtout une pièce sur l'enfermement, la victimisation, le temps et l'espace qui étouffent » (2000, p. 56), états d'âme mortifères renvoyant à ce qu'elle qualifie de "douleur innommable » (2000, p. 52), qui accable tout autant ses héroïnes romanesques et dont la condition de mutisme qui en résulte mérite un examen approfondi. Nous postulons donc qu'une approche de lecture orientée en fonction du concept de sujet subalterne, saisi en lien avec ses contreparties énonciatives et expressives, permettant d'ausculter deux romans farhoudiens selon les modalités du vouloir dire et du ne pas pouvoir dire chez des personnages situés à des moments distincts d'une trajectoire féminine saisie à rebours (allant de la vieillesse à la maturité), contribuera à enrichir notre compréhension de la poétique représentative de l'auteure ainsi que de son axiologie éthique. Comment les configurations du sujet subalterne se dessinentelles? Quels sont les procédés langagiers et les modalités rhétoriques qui entrent en jeu dans la prise de parole chez un sujet habituellement muselé? Et lorsqu'éclate cette voix féminine jusqu'alors réprimée, quels sont les aléas affectifs mis au jour? Quels seraient les enjeux impliqués dans la réception de la parole d'une subalterne? Afin de répondre à de telles questions, nous éluciderons un ensemble de concepts-clés développés par Spivak et par Cixous autour du silence et de la 
vocalité au féminin et présenterons notre cadre méthodologique, basé sur une approche énonciative étayée par les notions d'ethos (Maingueneau, 2004) et de praxis énonciative (Bertrand, 2000). Suivra l'investigation des deux romans farhoudiens qui composent notre corpus analytique.

\section{Du sujet subalterne et de la décapitation du féminin aux stratégies subversives de la voix}

D'inspiration poststructuraliste, les travaux de Spivak s'inscrivent dans le cadre précis du post-colonialisme et partent d'un concept qu'Antonio Gramsci (1980) a forgé pour décrire les réalités de groupes sociaux inférieurs (le sous-prolétariat et la classe paysanne), dont le témoignage fut exclu du discours historiographique officiel, plus précisément dans le contexte de l'Inde coloniale. La théoricienne, qui compte parmi les premières critiques à avoir révélé que la subordination dont souffrent les femmes découle à la fois de leur origine ethnique et de leur genre (Spivak, 2009, p. 53), s'intéresse à la notion de «silence» en tant que trait distinctif de l'altérité des femmes issues des classes défavorisées appartenant aux sociétés ayant subi une dominance coloniale ou impérialiste. Se penchant sur les raisons de l'«occultation asymétrique de la trace de cet Autre dans sa précaire subject-ivité » (2009, p. 37), Spivak établit que la marginalisation de ce groupe de femmes démunies relève d'une socialisation patriarcale qui empêche l'inscription de leurs récits à même les annales d'un discours public. D'où les difficultés chez plusieurs exégètes issus du monde occidental à cerner les attributs d'« une conscience-voix de la femme» (2009, p. 77), rendue muette, mais non 
insignifiante pour autant. Quant à Cixous, dans son étude intitulée "Le sexe ou la tête? » (1976), elle formule une critique des discours normatifs comme étant la propriété intrinsèque d'un régime phallique où la voix de la femme est exclue. Ce déséquilibre provient, d'après elle, de la prédominance de savoirs sociaux et philosophiques ancrés dans les logiques élaborées par Freud et Lacan, où le féminin ne figure pas à l'intérieur de l'ordre du symbolique : la femme est écartée « du langage, là où ça fait loi, exclue donc du rapport possible à la culture et à la règle de la culture » (Cixous, 1976, p. 8). La décapitation métaphorique des femmes relève donc d'une économie masculine libidinale; si celles-ci devaient parvenir à garder la tête, selon Cixous, cela ne serait qu' « à condition de la perdre, c'est-à-dire, dans le plus total silence et transformées en machines » (p. 6).

Dans sa discussion des limitations dont souffrent les femmes subalternes en ce qui concerne la mise au jour de leur subjectivité2, Spivak (2009, p. 25) fait intervenir deux concepts inhérents à la représentation, redevables à $\mathrm{Marx}^{3}$, pour souligner la complexité sous-tendant tout effort de transposition langagière d'expériences humaines touchées par l'exploitation. D'une part, elle convoque le concept de vertretung, terme allemand qui signifie parler au nom de quelqu'un en tant que représentant(e) ou mandataire, et d'autre part, celui de darstellung, notion qui renvoie à la représentation sémiotique ou esthétique, où il est question de

\footnotetext{
2 «Pour ce qui est du "vrai" groupe subalterne, dont l'identité consiste en sa différence, il n'y a pas de sujet subalterne irreprésentable qui puisse savoir et parler lui-même. » (Spivak, 2009, p. 48).

${ }^{3}$ Spivak (2009, p. 25) cite l'ouvrage de Karl Marx, Le Dix-Huit Brumaire de Louis-Napoléon Bonaparte (1851).
} 
parler de quelqu'un, dans un récit ou un portrait, par exemple. Spivak insiste sur ce genre de distinction pour montrer que les efforts de représentation du sujet subalterne par des observateurs-interlocuteurs, parlant soit en son nom (dans un contexte politique), soit à son sujet (lors d'un compte rendu, à titre d'exemple), masquent souvent des éléments de (re)construction chargés de présupposés ou d'une logique essentialiste. Elle illustre les enjeux de la représentation et de la non-représentation de l'autre au moyen de l'exemple du « sacrifice de la veuve» (Spivak, 2009, p. 77), soit le sati, qui en Inde renvoie à un rituel religieux d'immolation d'une veuve sur le bûcher de son défunt mari. Dans sa démarche, Spivak procède au démontage d'une multitude d'énoncés contradictoires - de provenance colonialiste, juridique, mythique et spirituelle - ayant fourni la justification de cette pratique archaïque à travers les époques, en amont et en aval de son interdiction ${ }^{4}$. Ces discours constituent un vaste réseau de manipulation épistémique qui, jouant sur les obligations et les responsabilités féminines, a eu pour résultat de transformer toute articulation de résistance en signe de consentement ${ }^{5}$. Les archives à cet égard sont catégoriques : «On n’a jamais affaire au témoignage de la conscience-voix de la femme » (2009,

\footnotetext{
${ }^{4}$ L'abolition du sacrifice des veuves entre en vigueur en 1829 (Spivak, 2009, p. 76).

${ }^{5}$ Pour démasquer le fonctionnement d'une stratégie patriarcale, où l'on prétend accorder à la femme le libre arbitre, Spivak évoque ce qu'elle nomme « la fabrique de la répression »: on fait la promotion de "l'être bon de la femme [woman's being good] » et, par voie de conséquence, « du désir de la femme bonne [the good woman's desire] », ainsi donc "du désir de la femme [woman's desire] » (Spivak, 2009, p. 81; le traducteur a placé les expressions en anglais de la version originale entre crochets dans le texte).
} 
p. 77) ${ }^{6}$. Devant le poids de tels mécanismes de réification sociale et de préemption psychique et verbale, Spivak demeure pessimiste à l'égard du pouvoir de réfutation chez les femmes asservies et conclut, en réponse à sa question éponyme, que le sujet subalterne féminin se trouve dépourvu de parole : "Il n’y a pas d'espace d'où le sujet subalterne sexué puisse parler. » (2009, p. 100) Il s'agit d'une position qu'elle a nuancée par la suite en expliquant que le silence du sujet subalterne découle d'une épistémè socioculturelle qui l'empêche d'accéder aux compétences rhétoriques ou scripturales permettant d'encoder son histoire au niveau d'un discours hégémonique (1999, p. 308). Qui plus est, reconnaissant que le sujet subalterne s'avère «irrémédiablement hétérogène » (2009, p. 45), elle souligne, s'inspirant de Pierre Macherey (1966, p.7), que ses modalités d'inscription signifiante peuvent adopter des formes non canoniques de discours susceptibles de passer inaperçues, et dont devrait tenir compte la critique. Le travail de celle-ci " consiste bien en effet ici à " "mesurer des silences". Ce peut être une description de l'acte d'"étudier, identifier et mesurer [...]

\footnotetext{
${ }^{6}$ Morton (2011, p. 73-75) précise qu'avant 1829 , le sati fut assimilé à un phénomène identitaire hindou à l'intérieur du cadre colonial, mais sa justification reposait sur des sources religieuses antiques mal interprétées. Les autorités du British East India Co. ont imposé la supervision de la pratique par des magistrats afin de s'assurer que les veuves consentantes agissaient selon leur liberté de choix. Mais les veuves se sont retrouvées devant deux discours contradictoires. D'une part, si le suicide s'appuie sur des raisons spirituelles, l'immolation se fondait sur des croyances erronées transmises de génération en génération; d'autre part, l'idée du libre arbitre, du point de vue légal, renvoyait à un concept inconnu dans le contexte en question : "Par l'inexorable production idéologique du sujet sexué qui l'accompagne [le sati], une telle mort peut être appréhendée par le sujet femme comme un signifiant exceptionnel de son propre désir, qui excède la règle générale relative à la conduite d'une veuve. » (Spivak, 2009, p. 83 ; l'auteure souligne) Il aurait été impossible de refuser l'immolation à l'intérieur d'un système de croyances où la femme devait se plier aux mentalités régnantes.
} 
la déviation" par rapport à un idéal irréductiblement différentiel.» (Spivak, 2009, p. 51; l'auteure souligne) En d'autres termes, dans son travail de décryptage à l'égard du sujet subalterne, la critique a tout intérêt à s'attarder sur la déviation de la parole vers d'autres modalités expressives, d'autres effets langagiers, qui, échappant aux codes prescrits et universels de représentation (situés à la fois à l'intérieur et à l'extérieur du contexte examiné), demeurent hautement signifiants (Spivak, 1999, p. 204). Jamais entièrement disjoint d'une assimilation d'aspects du discours dominant, le langage des subalternes se trouverait articulé différemment, à savoir dans des actes illocutoires singuliers, au moyen par exemple de manifestations gestuelles, visuelles ou acoustiques ${ }^{7}$.

Dans sa démarche théorique, Cixous abonde dans le même sens, du fait qu'elle présuppose une posture de résistance face aux mécanismes d'ordre patriarcal conduisant la femme à occuper un espace d'absence, isomorphe du silence. Cette posture s'érige contre ce qu'elle dénomme «l'empire du propre» (1976, p.11), qui renvoie au sens que lui donne l'établissement culturel dominant, où « règne l'homme en tant que propre» (ibid.), entraîné par une crainte de se voir exproprié de ses droits et de son pouvoir. Cette résistance ne pourrait se faire qu'au prix d'un bannissement des systèmes de

\footnotetext{
${ }^{7}$ Ashcroft, Griffiths et Tiffin (2007, p. 201) expliquent que, d'après Spivak, aucun acte de résistance chez un sujet subalterne ne pourrait être entièrement séparé «du discours dominant qui offre le langage et les catégories conceptuelles avec lesquels la voix subalterne s'exprime [...] et dans la plupart des cas le langage dominant ou le mode représentatif est approprié afin que la voix marginale puisse être entendue». Notre traduction : «from the dominant discourse that provides the language and the conceptual categories with which the subaltern voice speaks [...] and in most cases the dominant language or mode of representation is appropriated so that the marginal voice can be heard ».
} 
censure portant sur «toute tentative de dire qui serait une tentative féminine» (1976, p. 12). D’après la théoricienne, ce travail littéraire, loin du jugement, des interdits ou de la réprobation sociale, permettrait de transposer sous forme de signe engageant tant le signifié que le signifiant, des aspects primordiaux de la subjectivité au féminin. Dans cette perspective, Cixous établit clairement qu'une parole féminine est nourrie par l'inconscient, par ce qui passe par «la voix» (1976, p. 14), par une substance prélogique saisissable au niveau du vouloir sentir qui imprègne le vouloir dire, à savoir des composantes modales et modulatoires du pré-signifiant capables de se soustraire à une économie dichotomique associée à un positivisme logocentrique. Écrire au féminin reviendrait à retracer ce qui est coupé par le symbolique, «c'est-à-dire la voix de la mère, c'est faire passer ce qu'il y a de plus archaïque. La force la plus archaïque qui affecte un corps et qui est ce qui entre par l'oreille et qui atteint au plus intime. Ce toucher le plus intérieur fait toujours écho dans un texte de femme. » (ibid.) $)^{8}$

Adoptant des approches distinctes pour démasquer les procédés d'assourdissement qui obstruent la saisie des voix du sujet subalterne, Spivak et Cixous soulignent l'importance de

\footnotetext{
${ }^{8}$ D'après Stanford Friedman (1996, p. 32, note 1), la position de Cixous se rapprocherait d'une conceptualisation dénommée "gynésis», redevable à Jardine, qui a inscrit le féminin en tant que genre dans la critique que le poststructuralisme et le postmodernisme ont adressée aux approches humanistes en analyse littéraire. Cixous a mis en lumière l'importance de mettre à contribution certains effets discursifs du féminin au démantèlement des savoirs totalisants véhiculés par les «grands récits » (Jardine, 1991, p. 25). Les notions élaborées par Cixous et Spivak s'arriment avec d'autres éléments de discrimination qui vont au-delà du genre, soit la race, la géographie, la sexualité, la santé, l'âge et le niveau de scolarité, pour ne nommer que ceux-ci.
} 
l'intervention des instances critiques capables de repérer les infléchissements d'altérité au plan textuel. Dit autrement, sans les outils adaptés permettant de décoder les attributs rhétoriques parallèles ou subversifs de cette altérité, toute entreprise d'élucidation d'une subjectivité subalterne s'annoncerait forclose. Ces attributs, on l'a vu, sont emblématiques d'une poétique qui a tendance à valoriser la voix et ses inflexions en tant qu'extension du corps, ce vecteur génétique $\mathrm{du}$ sens constituant un cadre d'interrogation important pour explorer la subjectivité au féminin ${ }^{9}$. À cet égard, Luce Irigaray a montré que la voix peut facilement se modeler sur les propriétés informes du désir échappant aux typologies rigides : c'est «continu», « compressible», « dilatable », « diffusible » (Irigaray, 1977, p. 109), et surtout sa qualité fluide permet de «déborde[r] le sujet» qui d'habitude la fige et la « glac[e] dans ses catégories jusqu'à la paralyser dans son flux » (p. 111). Souscrivant à une telle pensée, Didier Anzieu établit la primauté de la voix par rapport à celle de la vision. Chez le nouveau-né, l'espace psychique se développe en premier lieu en fonction de l'écoute de la voix de la mère qui, tel un contact tactile, lui rappelle l'état fusionnel et bénéfique avec le corps de celle-ci durant le stade prénatal. D'après le chercheur, cet espace psychique constitue un miroir acoustique s'imposant avant le miroir optique que suggère Lacan, car l'acte de s'entendre soi-même et d'entendre autrui prépare le nouveau-

\footnotetext{
${ }^{9}$ Spivak rappelle la contribution importante de critiques soucieuses de saisir les traces ou les échos de la représentation du sujet subalterne là où on ne le cherche pas habituellement, notamment dans les interstices du texte ou à travers des données génétiques de la sémiosis. En conclusion, elle revient sur cette idée en disant que la «représentation n'a pas disparu [...]. L'intellectuelle femme, en tant qu'intellectuelle, a une tâche bien définie qu'elle ne doit pas désavouer d'un grand geste. » (Spivak, 2009, p. 103)
} 
né à ouvrir son espace psychique et à produire du sens avant même le processus conscient de son individuation (Anzieu, 1995, p. 195).

La pensée féministe valorise la voix dans la mesure où celle-ci est l'extension d'une corporéité prélogique qui, non scellée dans l'autosuffisance, s'ouvre à l'autre. Transcendant l'espace visuel, la voix, l'écoute et les régimes auditifs subvertissent les dispositifs représentatifs du féminin basés sur l'orientalisme et le regard masculin (male gaze), où s'exprime la crainte de la castration, et qui tendent à en offrir des images exotiques et réductrices (Mulvey, 1975; Bryson, 1986). Privilégier en revanche un régime auditif en tant que moyen de représentation permet de reconnaître comme signifiants le rythme, le souffle, le préverbal, la sphère émotionnelle du cerveau, attributs d'une subjectivité captée dans un déroulement qui débordent le visible. Julia Kristeva favorise une telle subjectivité, qu'elle a baptisée le régime sémiotic de la signification, d'origine psychique et corporelle, dont les propriétés, s'imposant en amont d'une signification catégorielle, imprègnent le sens ${ }^{10}$. Puisque la voix reflète une conscience en formation, elle permet de faire surgir des éléments d'une subjectivité réprimée ou archaïque et constitue, aux yeux de Cixous et d'Irigaray, une manière de sortir le sujet féminin de l'ombre. Aussi, compte tenu de sa résonance, la voix est le lieu naturel de la relation (Cavarero, 2005, p. 170), non seulement en raison de son évocation du rapport primaire nouant l'enfant à sa mère, saisi dans une indivision, mais aussi

\footnotetext{
${ }^{10}$ En proposant une sémanalyse, Kristeva (1969) a réhabilité l'instance d'un sujet producteur de la signifiance, s'ouvrant à la génétique, à l'inconscient et à la pluralité sensible travaillant le signe en amont de sa manifestation.
} 
parce qu'en tant que composante de base du mode communicationnel humain, elle interpelle l'écoute de l'autre : dire " je » présuppose une intersubjectivité, la présence d'un interlocuteur sous forme de «tu», de rappeler Benveniste (1966, p. 230-232).

Un cadre méthodologique souple inspiré du concept d'énonciation s'impose comme choix judicieux pour l'examen des configurations discursives du silence et de la voix chez Farhoud. Intégrant tour à tour l'émission et la réception de la parole féminine, une posture énonciative doublée d'une perspective d'ethos, concept qui provient de la rhétorique aristotélicienne, permet de tenir compte de la voix, de la parole et du type de discours adopté, aspects inhérents à la communication, par lesquels se forme l'adhésion du destinataire. En vertu de sa manière de représenter et de discourir, toute scénographie gère son rapport à cette vocalité fondamentale, à la fois origine énonciative et caution du dit énonciatif. Dans son prolongement du concept, Dominique Maingueneau accorde une grande polyvalence à l'ethos, dans la mesure où il permet de laisser présupposer au locuteur un caractère (traits psychologiques) et une corporalité (son inscription dans une réalité) renvoyant aux aspects sensibles, cognitifs et sociaux de la vocalité qui investissent le tissu discursif. C'est dire que la notion d'ethos « permet d'articuler corps et discours: l'instance subjective qui se manifeste à travers le discours ne s'y laisse pas concevoir seulement comme un statut, mais comme une voix, associée à la représentation d'un "corps énonçant" historiquement spécifié » (Maingueneau, 2004, p. 207). Ne posant aucune hiérarchie entre ce qui est dit et la manière de le dire, le théoricien évite ainsi toute rupture entre le texte et le corps, le monde représenté et l'énonciation, 
ce qui autorise à inscrire les œuvres examinées dans un contexte d'énonciation déterminé, rattaché aux idiolectes et aux sociolectes empreints de variabilité au plan d'une identité sexuée (Maingueneau, 1993, p. 139-144). Cela dit, il ne faudrait pas perdre de vue que les principes avancés par Maingueneau sont à envisager sous un angle abstrait en tant que présupposés de l'énoncé. Bhabha rappelle à cet égard (2007, p. 80) qu'une énonciation mimétique de la subjectivité s'avère au fond impossible: en vertu de la médiation de l'énonciation au moment de la mise en discours, on ne pourrait qu'identifier un certain nombre de repères ou de traces d'une voix-conscience au niveau de l'énoncé.

De même, en ce qui concerne la problématique de la représentation, on part du postulat que l'ethos présuppose l'intervention d'une praxis énonciative conceptuelle qui se charge de l'intégration des réalisables d'un usage idiolectal de la langue (la parole) aux universaux d'une structuration linguistique (la langue) : l'ethos recouvre une praxis énonciative qui concilie la genèse discursive, par l'intégration des produits de l'histoire en tant qu'effets et construits langagiers, et la convocation des universaux structurants de la narration (Bertrand, 2000, p.54). Dans cette perspective élargie, il est possible de tenir compte de la portée illocutoire de la langue et des modalités rhétoriques chez nos protagonistes féminins et, surtout, de reconnaître comme signifiantes certaines dimensions sensibles de leur parole. En clair, une telle approche permet d'évaluer les contraintes expressives et sociales auxquelles elles font face tout autant que les «vocalité[s] » (Maingueneau 2004, p. 207, l'auteur souligne) qui en émergent. 


\section{Conjurer le silence et l'indicible. Une voix monologique pour braver la honte}

En écrivant Le bonheur a la queue glissante (2004 [1998]), Abla Farhoud suit dans le sillage de Spivak, car elle s'est donné le défi de capter la parole d'une septuagénaire illettrée en arabe et aphasique en français. La romancière a choisi de se fondre «dans ce corps de femme, [afin d'] écouter [...] son rythme, ses silences [...], inventer sa parol. » (Chouinard, 1998). Évoluant depuis quarante ans dans une entière « dépendance » (Farhoud, 2004, p. 91) à autrui en ce qui touche aux aspects de la vie à l'extérieur du foyer, cantonnée dans un silence souvent résigné - «Je ne suis pas très bonne en mots. Je ne sais pas parler. Je laisse la parole à Salim.» (p. 14) —, Dounia s'avère pourtant dotée d'une performativité vocale exceptionnelle dont découle «la force du roman» (Montandon, 2006, p. 83). De quelle manière accède-t-on à la voix de ce sujet subalterne? À vrai dire, Farhoud parvient à faire surgir la vocalité chez ce personnage discret et pudique, ayant au cours de sa vie substitué ses talents culinaires aux mots ${ }^{11}$, grâce d'une part aux enjeux énonciatifs du récit, impliquant le monologue et la dimension acoustique de sa parole, et, en vertu, d'autre part, d'une exploitation de son usage de proverbes, catalyseurs d'aveux jusque-là indicibles.

À la fois narratrice homodiégétique et focalisatrice ${ }^{12}$, Dounia prend la parole au moyen d'un long « monologue écrit »

11 «Qu'est-ce qui est arrivé pour que mes mots se transforment en grains de blé, de riz, en feuilles de vigne et en feuilles de chou? Pour que mes pensées se changent en huile d'olive et en jus de citron? Qu'est-ce qui est arrivé?» (Farhoud, 2004, p. 16)

12 Chez Genette, le concept de voix narrative renvoie aux fonctions du narrateur qui, en plus de raconter, « organise, atteste et commente le récit, en 
(Rabaté, 1999, p. 78), dont la médiation énonciative relève de sa fille-écrivaine, Myriam. Le résultat aboutit à la transmission d'une oralité saisie sur le vif d'un personnage habitué à se taire, mais qui se livre dès lors à un destinataire posé en confident. Si son monologue constitue « un retour à soi [...], le vecteur d'une parole à la fois libératrice et transgressive » (Boireau, 1994, p. 166), il s'inscrit du même coup au niveau de l'intrigue dans un projet de collaboration avec Myriam qui aiguillonne chez sa mère le désir de la rétrospection ${ }^{13}$. Dounia s'engage ainsi dans un ultime espace d'interrogation, de remémoration et d'exploration de sa conscience, ce qui innerve son discours « de tension, de combat» (Rabaté, 1999, p. 79), qui se produit en amont de sa réclusion dans un hospice de vieillards, où elle abdiquera définitivement son droit à la parole. " Mes souvenirs me reviennent, toujours les mêmes, comme s'il ne me restait plus qu'une seule image pour chaque période de ma vie. [...] J'aimerais élargir mes images, pour mieux comprendre avant de mourir. "(Farhoud, 2004, p. 89-90)14 Le dialogisme enclavé sous-tendant ce monologue testamentaire présuppose un cadre d'écoute, l'accueil d'une tonalité vocale modulée par le vécu d'un personnage féminin souffre-douleur et orphelin de mère, marginalisé non seulement par ses pertes de repères culturels

relation à un narrataire », tandis que le mode présuppose « la distance que le narrateur entretient avec ce qu'il raconte [...] le point de vue adopté » (Mimouni et Robert, 2002, p. 597).

${ }^{13}$ Plusieurs critiques ont commenté l'ambivalence du roman, soulignant que la voix de Dounia résulterait d'une intervention énonciative de la part de Myriam, qui se chargerait de transposer en français les propos énoncés en arabe à l'aide du mode fictif. Voir Carrière et Khordoc (2006), Oore (2000) et Proulx (2004).

${ }^{14}$ Selon Erikson (1997, p. 65), cette étape du souvenir se caractérise par une médiation impliquant un désir d'intégrité confronté aux états de dysphorie, pouvant aboutir à un sentiment de cohérence et de complétude. 
et linguistiques dans sa transhumance entre le Liban et le Québec, mais surtout par les abus subis aux mains de l'ordre patriarcal. Pour cette raison, il n'est pas étonnant de constater que dans le monologue de Dounia s'intercalent des boucles narratives qui relèveraient d'un narrateur hétérodiégétique, associées aux interventions de Myriam ${ }^{15}$. La langue de Dounia qui « se délie » (p. 24), non sans tractations ni disputes (p. 117122), s'avère donc hétéroclite et fragmentaire, si bien que son décodage exige une lecture en contrepoint (Said, 1994, p. 6667) ${ }^{16}$ permettant de saisir « le grain de la voix » (Barthes, 1982, p. 237) de la septuagénaire qui résonne par-delà les interjections de sa fille. "La muette que je suis est capable de parler quand elle s'en donne la peine ou quand la peine devient trop grande et déborde » (Farhoud, 2004, p. 121).

Mimant une scénographie énonciative de l'autobiographie fictive, Dounia bénéficie d'une autorité non conventionnelle au vu du sujet subalterne et elle se rapprocherait ainsi d'autres héroïnes âgées, éprouvées par l'existence, s'exprimant sous forme monologique, notamment de Haggard de L'Ange de pierre de Margaret Laurence (2012 [1964]), engagée elle aussi dans un processus d'anamnèse avant son entrée dans une maison de retraite. Cette pratique

15 Il est possible de retracer un tel glissement vers une narration hétérodiégétique au moment de la description du « deuxième versant de la vie» (Farhoud, 2004, p. 11), de celle de l'éclatement de la guerre civile au Liban (p. 114-115) et, surtout, lors du dernier chapitre, lorsque Dounia est internée dans un hospice, où la narration à la troisième personne du singulier s'insère dans un récit en «je » et où une temporalité au présent cède aux verbes au conditionnel (p. 160).

${ }^{16}$ Nous traduisons ainsi "contrapuntal reading", qui recouvre une compénétration de récits, le dit et le non-dit, permettant de saisir le poids de la censure culturelle et circonstancielle dont souffre Dounia. 
rhétorique du monologue chez un personnage féminin, de souligner la critique, demeure cependant plus répandue au théâtre (Vigouroux-Frey, 1994; Geis, 1993) ${ }^{17}$ que dans le genre romanesque. À cet égard, les chercheurs qui se sont penchés sur le monologue écrit - à distinguer du monologue intérieur associé au courant de conscience, transmis par une narration à la troisième personne (Cannone, 2001, p.57-61; Cohn, 1981, p. 121-164) - offrent comme prototype du genre des romans où la parole revient aux personnages masculins (un ancien fonctionnaire, des écrivains anonymes, un juge ${ }^{18}$, lesquels possèdent une emprise discursive sur le monde, ne serait-ce qu'en vertu de leur capital logocentrique (Cixous, 1976). Farhoud, dont la carrière débute au théâtre (1993, 1994, 1997a, 1997b), a de toute évidence transposé au roman un mode d'adresse scénique permettant d'investir une allophone d'une prégnance vocale non équivoque du point de vue de l'adhésion du lecteur. Révélateur du « pouvoir du langage » (Rabaté, 1999, p. 92), ce procédé souligne l'inhérente originalité de la stratégie énonciative adoptée. Posé en miroir de soi, le monologue n'est pas uniquement révélateur de conscience secrète, il sert aussi à évoquer une image antérieure de la vieille dame, lorsque jeune fille, elle révélait des indices d'un talent oratoire (Farhoud, 2004, p. 15-16), mais qu'elle perd en tant qu'épouse de Salim, qui subvertit sa parole, impose sa supériorité par sa loquacité.

L'incorporation d'une modalité dramaturgique dans un roman basé sur la transmission d'un discours spontané,

${ }^{17}$ Chez Antonine Maillet (1990 [1971]), le personnage éponyme de La Sagouine est une monologuiste dans le domaine du théâtre.

18 D. Rabaté et B. Cannone citent les œuvres littéraires suivantes : Les Carnets du sous-sol de F. Dostoïevski, L'Histoire de la journée d'hier de L. Tolstoï, Le Bavard de L.-R. des Forêts et La Chute d'A. Camus. 
empreint d'oralité, permet de l'inscrire dans l'optique des actes du langage où les énoncés sont chargés de dimensions illocutoire et perlocutoire relevant d'un sujet psychosocial, dont le contexte de vie conditionne la prise de parole (Searle, 1972). À cet égard, le monologue de Dounia regorge d'incises qui trahissent sa peine, son indignation et sa véhémence, une dysphorie jusque-là inavouée qui éclate au moment de ses séances de travail avec Myriam : «[N]e me dis pas que je t'ai aidée à comprendre la vie comme une mère doit le faire. Tu m'as vue plier, tout accepter, me taire, est-ce un exemple de vie pour mes filles? [...] Ignorante, voilà ce que j'ai été, c'est la pire calamité. » (Farhoud, 2004, p. 120) ${ }^{19}$ Des éléments à la fois morphosyntaxiques et acoustiques de sa narration, relevant du signifiant, dévoilent l'ethos du sujet subalterne, car ils sont en consonance étroite avec les signifiés, ce qui laisse transparaître une affectivité souterraine liée aux aléas vécus. À titre d'exemples, le récit de Dounia abonde en modalités exclamatives symptomatiques de l'exaspération: "Même le prophète Mouhammad ne saurait répondre à toutes les questions que Myriam me pose!» (p. 24), ou «Et pourquoi n'aurais-je pas le droit de philosopher, j'ai vécu les trois quarts d'un siècle! » (p. 79). Le lecteur est par ailleurs interpellé par des questions rhétoriques offertes en cascade où s'élève une voix courroucée :

Comment ai-je pu le laisser partir? Est-ce que j'étais consciente de la situation? Est-ce que j'ai essayé de le convaincre d'attendre que les enfants grandissent? Et Salim, [...] comment

\footnotetext{
${ }^{19}$ Voir aussi l'expression de la douleur à l'égard de son fils : «Quand mon fils tombe en enfer, c'est plus fort que moi, [...] je tombe avec lui, j'éclate en mille morceaux et ma peine devient déraisonnable, mille jarres bourrées se fracassent, comme si j'étais la seule mère au monde à avoir un enfant malade... » (p. 133-134)
} 
a-t-il pu tout laisser sans se poser de questions? Et rendu làbas, comment a-t-il pu jouer au riche, dilapider [...] l'argent que les enfants avaient gagné tout autant que lui? (p. 90)20

Les répétitions, chez Dounia, sont souvent un moyen de revisiter le bouleversement causé par une crise sans issue : « Où aller? Où aller avec mes enfants et enceinte jusqu'aux lèvres? [...] Où aller? Où peut aller un clou sans tête?» (p. 144). À d'autres moments, les mots en italique miment une emphase vocale, traduisant ainsi ce qui ne saurait s'exprimer en langage étoffé :

Émigrer, s'en aller, laisser derrière soi ce que l'on va se mettre à appeler mon soleil, mon eau, mes fruits, mes plantes, mes arbres, mon village. Quand on est dans son village natal, on ne dit pas mon soleil, on dit le soleil, et c'est à peine si on en parle puisqu'il est là, il y a toujours été là, on ne dit pas mon village puisqu'on l'habite... (p. 42)

Les points de suspension divulguent chez la protagoniste une pensée en formation se rapprochant d'un indicible névralgique: «Moi?... Le plus important?... Mes enfants ... La chose qui a été la plus importante [...]? C'est... attends... laissemoi réfléchir... je dirais que c'est d'avoir émigré. Oui. [...] Mais si j'y pense un peu plus longtemps, je me dis que non, ce n'est pas ça qui a changé ma vie... C'est... » (p. 121) ${ }^{21}$ Et les ellipses sont les coefficients de non-dits et d'aveux :

Comment mettre de l'ordre quand tout peut éclater à n'importe quel moment?... Toute ma vie... non, pas toute ma vie... depuis

\footnotetext{
${ }^{20}$ Un autre exemple : «Est-ce que ça va revenir cette année? Quoi faire pour que ça ne revienne pas? Comment faire? Pourquoi? » (p. 131)

${ }^{21}$ Voir également : «[q]uand je mourrai... je m'ennuierai de mes petitsenfants... et de mes enfants aussi... je ne sais pas s'ils penseront à moi quelquefois... C'est bon, le silence... la paix et le silence. J'aurais dû me faire religieuse... chanter et prier et écouter le silence... mais je n'aurais pas eu de petits-enfants. » (p. 22)
} 
mon mariage, j'ai toujours essayé de prendre sur moi, de patienter, de me cacher dans le silence, de me dire ça va passer... [...] Ça n'est jamais passé. [...] J'ai attendu longtemps pour élever ma voix. Trop longtemps. (p. 133)

Chargées de confidences, les interpellations s'adressent souvent à son fils malade : "Mon enfant, quelle mauvaise étoile t'a conduit jusqu'à nous? Mon fils tant aimé, en naissant tu t'es trompé de parents. [...] Mon fils, plus je ressasse nos vies, plus je les tourne à l'endroit et à l'envers, plus je sais que tu t'es trompé de parents.»(p.138) De même, certaines formules enfantines monosyllabiques rendent bien des tentatives limitées d'expression en français, «David m'a dit [...] : “Tu es triste, sitto?" J'ai dit : "Non, moi pas triste, moi vieille, pour ça." » (p. 121) Relevant, selon Cixous, de "la chair de la langue» (1976, p.14), tous ces procédés stylistiques expriment de manière variable la dimension pathémique, sensible, de la parole de Dounia, l'«exaltation d'une voix» (Hubier, 2003, p. 106). Ils sont les sites de divulgation d'une subjectivité qui se cherche et par laquelle s'affirme une agentivité qui compense chez cette subalterne une lacune de ressources discursives.

Comme nous l'avons signalé, une caractéristique importante du monologue de Dounia réside dans son recours constant aux proverbes. Aux yeux de certaines critiques, cette tendance à émailler son discours d'adages et de dictons confirmerait ses inaptitudes communicatives (Boustani, 2003; Oore, 2000). Nous postulons que l'emploi de proverbes se rapprocherait davantage d'un procédé de ré-énonciation que Dounia adopte non seulement pour évoquer son héritage, mais aussi comme stratégie rhétorique de subversion et de 
véridiction $^{22}$. Rappelons qu'est proverbe un énoncé concis inscrit dans une tradition qui exprime une vérité fondamentale transmise d'une génération à l'autre (Dagher, 1994). Nombre de chercheurs retracent ses origines dans la civilisation grécoromaine antique ainsi que ses usages qui remontent au Livre des Proverbes et aux genres brefs de la littérature du Moyen Âge et acquiescent qu'il se relie au folklore et à la paysannerie, où prédomine une culture d'oralité (Emeto-Agbasière, 1986; Soriano [en ligne]). Objet de la parémiologie, les proverbes sont dotés d'une intemporalité les rapprochant des contes et des légendes (Greimas, 1970, p. 313) et, au Liban, de signaler Michel Feghali (1938, p.xi), ils font partie d'une littérature populaire qui a imprégné les rituels communautaires. Le chercheur cite le phénomène des joutes organisées à partir de la récitation publique des proverbes, pratique restée vivante dans bien des villages libanais, dont remonte une tradition du chant fondée sur la « zajal » (Haydar, 1989, l'auteur souligne) ${ }^{23}$.

Confirmant son appartenance à une collectivité soudée par une forte tradition orale, un monde « imagé, poétique, plein de raccourcis fulgurants » (Montandon, 2006, p. 84), l'emploi de proverbes chez Dounia reviendrait à illustrer une autre composante de la langue parlée, car la profération d'un proverbe implique non seulement une motivation rythmique, syntaxique et lexicale, mais aussi une visée prosodique qui se dénote par un changement d'intonation (Meschonnic, 1976,

\footnotetext{
22 La véridiction est un processus intra-discursif lié aux instances de l'énonciateur et de l'énonciataire et où interviennent le faire persuasif de l'un et le faire interprétatif de l'autre (Bertrand, 2000, p. 268).

${ }^{23}$ Dounia rappelle que Salim est nostalgique de l'époque où il bénéficiait d'un auditoire à ses histoires racontées « sur la place du village » (p. 40) au Liban, ce qui n'existe pas dans son pays d'adoption.
} 
p. 427). Plus significatif encore, les proverbes sont intégrés dans le monologue de Dounia en tant que dispositifs de représentation identitaire (Sniader Lanser, 1992, p. 115) et font partie de l'unique héritage reçu de sa mère, décédée alors qu'elle était en très bas âge. Il s'agit notamment de deux proverbes libanais réitérés par sa grand-mère, à savoir, « [n]e laisse jamais passer les instants de plaisir; pour rassasier ton corps, un rien suffit» et "[i]l n'y a pas de souffrance que le sommeil ne sache pas vaincre» (Farhoud, 2004, p. 28), que Dounia lègue à son tour à Myriam. Renvoyant à la primauté signalée par Anzieu de la voix dans l'espace fusionnel liant l'enfant à sa mère, cette filiation maternelle eu égard aux proverbes n'est pas anodine. Que Dounia invoque sa mère lorsqu'elle dénonce les limitations qu'une société traditionnelle impose à la femme, reflétée par l'axiologie des proverbes, cela ne l'empêche pas d'explorer autrement leur soubassement mémoriel. Une telle hypothèse se soutiendrait dans la mesure où les proverbes transmis sont indissociables de la figure « d'un petit carré de dentelle» (p. 29) convoité par la jeune Dounia, reçu de sa voisine le jour de la mort de sa mère, acte qui, en provoquant de la joie, lui permet d'atténuer sa peine. Pratique féminine, ayant ses antécédents en Orient, la fabrication de la dentelle se relie du point de vue symbolique à la création en autant qu'elle présuppose un genre de tissage qui renvoie à «tout ce qui commande ou intervient dans notre destin » ${ }^{24}$, ce qui n'exclut pas l'idée que tisser permet en même temps de « créer des formes nouvelles » (Chevalier et Gheerbrant, 1982, p. 1097-1098) : «Tisser ne signifie pas seulement prédestiner

\footnotetext{
${ }^{24}$ Chevalier et Gheerbrant précisent que divers éléments liés à l'acte de tisser - tissu, fil, métier, instruments servant à filer ou à tisser (fuseau, quenouille) — sont tout autant « symboles du destin » (1982, p. 1097).
} 
[...], mais aussi créer, faire sortir de sa propre substance, tout comme le fait l'araignée, qui bâtit sa toile d'elle-même » (Eliade, 1964, p. 159) ${ }^{25}$. Greffée aux proverbes, une telle notion d'invention féminine empreinte du « registre du maternel » (Robin, 2003, p. 56) laisse entrevoir leur fonction structurante au sein du monologue. Dounia s'en sert certes comme moyen d'autodéfense, mais ils font souvent double emploi puisque, si leur récitation nécessite la mise en œuvre d'un acte de langage idiolectal, celui-ci est à la fois déclencheur d'un approfondissement psychique infléchi par un héritage maternel réclamé tacitement.

Associés au geste du tissage, les proverbes offrent en effet des linéaments au récit de Dounia, leur rôle d'encadrement énonciatif reflété et par le titre et par le lexique retrouvé à la fin du roman, recouvrant la plupart des proverbes cités. Appuyant l'idée que l'emploi de proverbes relève d'une stratégie discursive, Bakhtine rappelle qu'ils appartiennent à un discours folklorique retransmis et représentent un ensemble d'énoncés où convergent des éléments centrifuges et centripètes (1981, p. 272); lorsqu'un énonciateur se les approprie, il les pourvoit de ses intentions uniques de même que des inflexions de sa propre voix (1981, p. 293) ${ }^{26}$. On observe les enjeux de ce genre de ré-énonciation chez Dounia. Si elle avoue qu'ils lui ont permis de dissimuler certains aspects douloureux de son passé, lui épargnant de les «revivre» (Farhoud, 2004, p. 30), à d'autres occasions, elle les invoque sciemment pour communiquer son opposition ou pour ôter son masque. À titre d'exemple, Dounia conteste l'intrusion de Myriam dans

\footnotetext{
${ }^{25}$ La citation d'Eliade est tirée de Chevalier et Gheerbrant (1982, p. 1098).

${ }^{26}$ Les renvois à Bakhtine sont empruntés à Bacchilega (1995, p. 89).
} 
l'interprétation de sa vie, dans l'édulcoration des faits. Aux insistances de celle-ci, elle réplique : " "Si tu n'es pas fatiguée de m'entendre, moi, je suis fatiguée de parler.”. [...] Elle n’a pas voulu comprendre. Elle a recommencé avec ses questions. Alors, je lui ai sorti le proverbe qu'elle déteste : “Celui qui est né est pris au piège et celui qui meurt se repose...” »(p.119) Prononcé deux fois, ce proverbe devient un catalyseur d'aveux, le manifesté d'une douleur enclavée qui resurgit, permettant à Dounia d'approfondir sa confession, d'admettre à sa fille sa soumission au destin. Elle parvient ainsi à exprimer ses regrets, son aversion de soi eu égard à son état de femme asservie en plus de l'enjoindre de taire la maladie de son fils, Abdallah ${ }^{27}$. Ce genre d'irruption verbale divulguant tout un substrat d'expériences refoulées et sensibles montre dans quelle mesure Dounia se sert des proverbes non seulement pour déstabiliser ses interlocuteurs, mais aussi pour entamer sa propre autocompréhension, prouvant de la sorte que son allure de femme sage « que plus rien n'ébranle» (p. 91) est un leurre.

Ce phénomène de tissage vocal qualifie l'espace des proverbes dans le cas de la honte. Renvoyant aux sentiments d'embarras, d'indignité ou d'humiliation générés par des états ou des actes soumis à l'opprobre public, Johnson et Moran (2013, p. 3) précisent que la honte demeure une émotion à prédominance féminine, non seulement en raison du corps qui incarne traditionnellement la honte, mais aussi dans la mesure où celle-ci structure trois aspects de l'identité féminine,

${ }^{27}$ Dounia parlant à Abdallha : «Et ta sœur Myriam qui voudrait que je parle de toi, à haute voix. » (p. 138) 
individuel, familial et culturel ${ }^{28}$. Une première source de honte chez Dounia revient à son état d'analphabétisme dont ses propres filles sont les repoussoirs, Myriam écrivaine et Kaokab professeure. Or on dénote dans un premier temps une réaction de déni, comme si sa honte ne pouvait se confronter qu'en passant par deux disqualifications sous forme de proverbe et de dicton, ce qui a pour effet d'amoindrir l'importance de savoir lire et écrire. En réfléchissant à l'incidence de l'éducation sur ses enfants, elle remarque que les gens instruits emploient un surcroît de mots pour exprimer ce qui pourrait se dire plus simplement: «[l]e meilleur des mots, un seul...» (Farhoud, 2004, p. 25), suivi par: «[n]e nous manque que ce que l'on a connu » (p. 29), réplique donnée à une question posée sur son illettrisme. Or ces réactions d'apparence superficielle ne sont pas sans retentissement. Elles ont pour effet de susciter des émotions jusque-là inavouables qui appuient une position contraire, car suivra une anecdote où Dounia explique les conséquences néfastes de son analphabétisme. Durant les premiers mois de son immigration au Canada, au bord du gouffre, elle désire écrire à son père au Liban pour lui demander de venir la chercher avec ses enfants. Elle subtilise une missive que Salim lui a adressée et, dans un «barbouillage » enfantin (p. 32), essaie de tracer « les quelques lettres que je savais... je ne savais pas écrire mon nom. J'avais trente ans, j'avais cinq enfants, bientôt six, et je ne savais même pas écrire mon nom... » (ibid.) À cette demande d'aide bâclée, jamais il n'y a eu de réponse. D’ailleurs, Dounia revient sur la béance créée par l'absence de secours maternel dans les

\footnotetext{
${ }^{28}$ Farhoud (2015, p. 50) abonde dans ce sens : «aayab... “[1]a honte”, mot pesant, chargé de mille sens, qu'on apprend presqu'à la naissance quand on est une fille, et beaucoup plus tard quand on est un garçon. »
} 
circonstances en question, «[c]ette lettre que j'ai tant désiré écrire, c'est à ma mère qu'il m'aurait fallu l'envoyer. Ma mère aurait compris, j'en suis sûre.» (p. 33), rappelant l'écho maternel qui retentit par l'entremise des proverbes. Reste qu'une telle confrontation à son analphabétisme permet d'exhaler sa colère et sa honte; sa riposte est d'ordre éthique, car en tant que subalterne ayant subi une sujétion similaire à celle de sa mère, illettrée elle aussi (p. 27), elle doit trouver le courage pour admettre qu'elle « aurai[t] tellement aimé savoir écrire » (p. 31), et que " [n]e pas savoir lire et écrire [lui] a beaucoup manqué » (p. 30). Si cet affrontement avec sa propre honte se clôt par un dicton qui contredit les énoncés précédents, soit « $[\mathrm{u}] \mathrm{n}$ prisonnier qui sait lire et écrire n'est pas en prison » (ibid.), cette ré-énonciation est teintée d'un certain blâme adressé à son père qui n'a rien facilité pour qu'elle puisse développer sa littéracie. On se souviendra que Dounia rompt ses études élémentaires en raison de la frayeur qu'elle ressent devant un instituteur sévère. Si son père donne l'impression de la soutenir dans son apprentissage de la lecture par après, il ne le fait que du bout des lèvres, lui disant: «[p]rends un journal et lis » (p. 81). On renvoie ainsi à une situation de délaissement souvent caractéristique du vécu des jeunes filles et le regret qu'il suscite resurgit, quelque soixante-dix ans plus tard, comme sentiment vif d'humiliation ${ }^{29}$. Mais Dounia n'est pas sans armes; rétorquant à l'invective paternelle, fût-ce rétrospectivement, par l'exclamation, «facile à dire!» (p. 86),

\footnotetext{
${ }^{29}$ Voir Le Liseur (Schlink, 1996). En raison de sa honte, Hanna ne peut avouer son analphabétisme lors d'un procès, ce qui l'aurait innocentée d'une accusation de complicité dans l'extermination d'un groupe de femmes juives lors de l'évacuation du camp d'Auschwitz. Elle est inculpée et doit purger une peine importante.
} 
elle montre combien sa propre exploration d'un certain nombre d'adages, en tant que formes de mimétisme et de recatégorisation des discours établis, lui permet de déstabiliser l'emprise patriarcale sur sa condition de femme ${ }^{30}$.

Ce genre de dénonciation des mentalités rétrogrades s'approfondit au moment d'affronter une seconde grande source de honte reliée, elle aussi, à une formule proverbiale : «Laisse ton mal dans ton cœur et souffre en silence; le mal dévoilé n'est que scandale et déshonneur.» (Farhoud, 2004, p. 143) Il est question d'explorer une blessure encore plus enfouie, qui remonte à un incident d'abus conjugal, perpétré par Salim, alors que Dounia, durant la trentaine, se trouve de nouveau enceinte, une scène humiliante à laquelle assiste son père, le prêtre du village, qui avalise l'acte commis (p. 147-148). Provoqué par ce souvenir douloureux, l'éclatement verbal de Dounia représente une instance de confession qui tire son urgence du fait qu'il lui importe de rompre le silence peu avant de succomber à une existence végétative à l'hospice. L'expression de cette " conscience-voix de la femme » (Spivak, 2009 , p.77) est enfin possible, car le cadre du monologue présuppose une réception sans jugement, ce qui aurait été impensable dans l'espace communautaire de sa jeunesse ${ }^{31}$. On

\footnotetext{
${ }^{30} \mathrm{D}$ 'après Bhabha, il s'agit d'une forme de mimétisme du pouvoir patriarcal qui, par son altérité, déstabilise le père et l'encourage à se reconnaître dans celle qu'il domine, permettant ainsi à la fille de subvertir son autorité. «La menace que fait peser le mimétisme est sa double vision qui, en dévoilant l'ambivalence du discours [patriarcal], démolit aussi son autorité. » (2007, p. 152; l'auteur souligne).

${ }^{31}$ Khannous (2000, p. 56) cite l'exemple donné par Spivak d'une hindoue de dix-sept ans, B. Bhaduri, qui s'est suicidée en 1926 afin d'échapper aux représailles vu son incapacité à compléter une mission d'assassinat. Elle a attendu ses règles pour montrer que sa mort n'était pas le résultat d'une grossesse illicite ni d'une passion illégitime. Se suicidant dans de telles
} 
saisit tout le poids de la souffrance causée par le refoulement de cette dégradation qui revient la hanter, se trahissant au moyen de sa diction. La reprise de la locution, "il y a des choses», martelée comme un anathème, dévoile la profondeur de sa déchirure et son appréhension à la confronter :

Il y a des choses que l'on ne peut pas dire [...]. Il y a des choses qui remontent malgré soi, comme une vomissure, [...] il y a des violences que l'on ne peut pas pardonner [...]. Il y a des choses qui ont le poids de toutes nos peurs rassemblées, de toutes nos lâchetés. Il y a des choses que l'on ne peut ni raconter ni dire à voix basse tant on en a honte. Il y a des hontes qui ne peuvent s'apaiser avec le temps, que l'on ne peut se pardonner, ni oublier. Des hontes qui restent intactes comme si on venait de les vivre. (Farhoud, 2004, p. 147)

Cette litanie-diatribe révèle combien Dounia s'en veut de ne pas avoir protesté contre les excès d'une autorité patriarcale fondée sur une collusion entre son mari qui la brutalise et son père qui la maudit, alors qu'elle est jeune mère de famille. Se répercute paradoxalement sur celle-ci la faute d'avoir franchi les limites de son rang subordonné, lorsqu'elle demande à son mari de ne pas quitter le foyer familial, alors qu'elle s'apprête à accoucher. Salim perçoit cette requête de soutien comme une déclaration de faiblesse qui l'atteint dans son orgueil et, du haut de son cheval, il lui assène un coup de botte qui lui « fen[d] la

conditions, elle accomplit « une inversion de l'interdit qui refuse à la veuve qui a ses règles le droit de s'immoler» (Spivak, 2009, p. 228). Khannous précise que ce acte radical, un affront à la pratique traditionnelle du sati, ainsi que la lettre d'explication laissée en tant que témoignage d'une parole subalterne, n'ont eu aucun retentissement, ni dans la famille de B. Bhaduri ni dans son milieu, parce qu'il n'y avait aucun espace réceptif à son discours. Spivak souligne que la subalterne n'est pas en situation de parole là où elle est objet de discrimination, mais que cela ne devrait pas la décourager de parler en son nom dans d'autres contextes (Spivak, 1999, p. 306), comme cela se produit chez Dounia. 
lèvre » (p.142). Puisque cet acte d'une violence extrême vise expressément la bouche de Dounia, ses lèvres, sa langue et ses organes oratoires, on observe combien oser parler chez une femme dans le contexte culturel en question, oser enlever une seule fois sa "muselière" (p.143), se perçoit comme une offense provocatrice à l'endroit de l'honneur masculin. Une attitude similaire se détecte chez le père de Dounia, car celui-ci, au lieu de la défendre, la condamne, ensemble avec sa mère : « Il maudissait ma naissance, mon être tout entier, le jour et la personne qui m'avaient donné la vie.» (p. 142) Dounia n'a ni divorcé de Salim, ni critiqué la trahison de son père, car elle se sentait obligée de se plier au code de l'honneur familial, auquel fait écho l'archi-proverbe énoncé, l'enjoignant au contraire de souffrir en silence afin d'éviter le scandale et le déshonneur (p. 143). À l'instar de sa mère comme de toutes les femmes "pétries de ces mots » (p. 150), Dounia essuie son humiliation, réprime sa colère sans montrer la moindre indignation ${ }^{32}$. «Trahir ce père qui venait de me trahir, le faire vivre dans la honte d'une fille sans mari et sans toit, j'en étais incapable. » (p. 143) Sa situation d'asservissement psychologique évoque une mentalité répandue dans l'aire de la Méditerranée voulant que la sauvegarde de l'honneur collectif, ancrée dans des sociétés fondées sur des clans, passe en effet par la respectabilité de la femme, objet de surveillance masculine accrue (Bourdieu, 2000). Elle subit donc une double misogynie, une double violence, conjugale et paternelle, qui déclenche chez elle des sentiments mortifères de honte et de culpabilité qui l'ont paralysée dans ses tentatives de porter plainte: «Parce

\footnotetext{
32 Jeune femme, Dounia incarne, à l'avis de Verduyn (2001, p. 299-304) citant $\mathrm{N}$. Brossard, une mère patriarcale du fait qu'elle reste pour la plupart cantonnée dans un rôle de reproduction.
} 
que ce père et toute sa communauté d'hommes, et de femmes aussi, nous ont appris à plier, à nous taire, à ne rien dévoiler, à avoir honte, à tout endurer. " (Farhoud, 2004, p. 143) ${ }^{33}$. De plus, ayant encaissé le mal, elle se blâme d'avoir laissé son conjoint «broyer la famille entière» (p.152), étendre sa barbarie sur Abdallah, à jamais marqué par des séquelles psychologiques (p. 153). Cette séquence lancinante d'aveux se clôt sur un effet prosodique fondé sur une répétition adverbiale ("comment dire»), doublée de l'accumulation de ses torts, processus révélateur de sa recherche acharnée de moyens langagiers capables de briser la chape de silence sous laquelle elle a vécu :

Comment dire à ma fille que la maladie de son frère [...] n'est qu'une malchance [...]. Comment m'avouer que c'est mon propre manque de dignité qui a détruit ma famille [...]. Comment dire toutes les violences que j'ai subies [...], comment parler de ma honte, de ma résignation, de ma rancœur, de mon amertume et de ma haine, de ma lâcheté, sans vouloir mourir [...]. Comment dire la vérité que j'ai cachée [...], comment dire que mon père est un lâche [...]. Comment dire que je n'ai plus aucun respect pour mon mari [...]. (p. 153)

En dépit de ses questions laissées sans réponses, c'est en vertu de son degré de force viscérale que ce segment du monologue de Dounia, telle une catharsis dramatique, a pour résultat de conjurer son mutisme et sa haine de soi. Fort d'une vocalité contestatrice qui éclate in extremis, le sujet subalterne se transforme dès lors en un sujet agissant qui tente de réhabiliter une identité meurtrie (Carrière et Khordoc, 2006,

${ }^{33}$ L'éclatement de son sentiment intense de culpabilité s'accompagne d'une invocation de sa mère: "Parce que ce père, qui allait jusqu'à insulter la mémoire de ma mère qui avait enfanté une fille qui se laissait fendre la lèvre, nous avait appris à le respecter, à l'honorer, à respecter nos frères et notre mari, à dépendre du soutien des hommes. » (p. 143) 
p.123-124). Qui plus est, subvertissant le proverbe emblématique qui fait référence à la mentalité rétrograde dont découle sa détresse, Dounia signale que la conclusion de son récit, dont la régie incombe à Myriam, consisterait en une version transcendée des faits, car une réconciliation avec Salim lui est pour ainsi dire inconcevable ${ }^{34}$.

\section{Entre réclusion et deuil.}

\section{Vers un accomplissement de soi par des paroles en acte}

Dans Splendide Solitude (2001) Farhoud élargit les contours du sujet subalterne en réorientant la question de l'aphonie féminine dans le sens d'une aliénation identitaire et sociale, ce que nous observons chez une narratrice innommée, la cinquantaine entamée, confrontée par un cumul de pertes et de deuils. Celle-ci, que nous désignons par la majuscule N. se séquestre chez elle dans le but d'exorciser sa détresse et de comprendre enfin «quelle a été [s]a vie» (Farhoud, 2001, p. 33). En dépit de son statut de bourgeoise cultivée, l'héroïne incarne les traits de sujétion dont parle Spivak du fait qu'elle a évolué dans une entière dépendance affective vis-à-vis d'un compagnon artiste. Négligeant ses propres talents d'interprète à la faveur de la carrière de son conjoint, elle s'est laissée enliser dans une idéologie patriarcale de soumission. «C'est lui qui montait sur scène, c'est moi qui restais dans les coulisses. Je suis restée dans les coulisses de ma vie sans jamais me mesurer, sans jamais me mettre en avant. Par amour? Par facilité? Ou par

\footnotetext{
${ }^{34}$ Le lecteur saisit l'ironie de la séquence finale lorsque Salim explique à Dounia: "Abdallah est l'envoyé du destin pour nous faire comprendre quelque chose. Il faut que tu pardonnes, Dounia, il le faut. » (p. 160)
} 
peur?» (Farhoud, 2001, p. 187) Au prix d'une introspection prolongée, elle constate combien son existence fut dictée par des comportements hypocrites et lâches; son altruisme apparent lui a permis de camoufler un "manque d'intérêt envers [elle]-même» (p. 189) et, plus grave encore, son silence «n'était pas seulement [s]on refuge, mais le lieu de toutes [s]es illusions » (p.190). Dit autrement, son penchant marqué pour la conciliation et le charme lui a fait adopter la posture et la mentalité de «l'ange domestique », dont a parlé Virginia Woolf (1970 [1942]) ${ }^{35}$, ce qui a fini par la détourner de toute analyse pouvant l'acheminer vers l'épanouissement. $\mathrm{Vu}$ son investissement complet dans la personne de son conjoint, sa désorientation au moment de la rupture se vit en termes d'exil, à telle enseigne qu'on est tenté d'établir des points de comparaison entre la vie de couple et une situation coloniale. «Après son départ, je ne savais plus qui j'étais. J'ai été projetée dans une ville étrangère, sans réseau, sans lien, sans appartenance [...]. Une implosion. [...] Mon corps avait mille morceaux disloqués. Ce n'était pas douloureux. C'était au-delà de la douleur.» (p. 21) Son sentiment de désintégration identitaire à la suite de la brisure se rapprocherait en effet d'une expérience de déracinement culturel: «Je venais de perdre ce qui était devenu ma patrie : la musique de celui que j'aime. Apatride, je le suis devenue, sans changer de maison. » (p.35) Isolée dans une mélancolie dont elle ne se remet pas, N. languit, se prive d'un exutoire public. Bien que témoin des revendications féministes de son époque, renvoyant aux années 1970 et 1980, elle vit en marge de ses consœurs: elle se retrouve sans projet autonome, sans capacité de transformer sa

35 Il s'agit de notre traduction de l'expression, «The Angel in the House » (Woolf, 1970 [1942], p. 236). 
colère « en action » (p. 170). Tandis que ses amies protestaient et « jetaient leurs soutien-gorge sur la place publique » (p. 51), elle se contentait dans son rôle traditionnel d'épouse et de mère tout en remplissant ses fonctions de « rentière » (p. 91) qui lui assurent la sécurité au plan financier.

Or le roman divisé en 57 chapitres, recoupant autant d'entrées dans un journal en train de s'écrire, représente une tentative impérative de divulguer son histoire, celle d'une femme bernée, assujettie au plan de sa vie intime, dont les drames pourraient passer inaperçus. En inscrivant sa bataille solitaire et tumultueuse, menée « entre moi et moi » (p. 22), à l'intérieur d'un cadre post-féministe, qui a eu tendance à évacuer le privé des revendications féministes généralement orientées vers l'obtention de la parité professionnelle (Steinem, 1992, p. 3-17), N. brave une sorte de censure implicite, ce qui rend son témoignage éclairant: " J'ai dit adieu à tout ce qui me divertit de moi-même, pour retrouver par moi-même et en moimême la flamme de vie [...] qui tout au long de ma vie a été allumée par les autres. [...] Le chemin de moi à moi est si long et ardu.» (Farhoud, 2001, p.146) Qualifiant l'énonciation du roman, ce dialogue intradiscursif permet à l'héroïne non seulement de se scinder ${ }^{36}$, de se distancier de son double angélique (Woolf, 1970 [1942], p. 238), mais aussi de multiplier les perspectives sur soi, de donner chair à sa propre révolution intérieure (Steinem, 1992), afin de conquérir une altérité refoulée et inconsciente, trajectoire au bout de laquelle elle parvient à (re)naître et à faire résonner sa propre voix, son

\footnotetext{
36 Autant rhétorique qu'éthique, ce procès permet d'explorer deux subjectivités inhérentes au «moi», celle d'un sujet d'énonciation situé au présent du discours et celle d'un sujet d'énoncé renvoyant au passé narratif.
} 
« cri » (Farhoud, 2001, p. 49), sous forme d'une performativité vocale livrée publiquement.

Splendide Solitude s'offre à bien des égards comme un canevas préparatoire à cette prestation se déroulant en temps réel devant un groupe d'auditrices-amies. C'est le récit d'une descente au purgatoire où l'auto-auscultation emprunte les traits d'une métanarration, d'un voyage sans boussole, caractérisé par l'évocation d'une désorientation et la description des efforts déployés pour sortir de l'aliénation, processus qui participe de la métaphore coloniale précitée. « Le passage à l'autre, je ne l'ai jamais fait. Et je ne pourrai le faire tant que je ne rapatrierai pas $m a$ constitution et les lambeaux effilochés et toutes les parties de moi oubliées qui ont perdu leur souveraineté en route. Recouvrer mon autonomie ne se fera qu'à ce prix.» (p. 189; l'auteure souligne) ${ }^{37}$ La forme du journal intime convient bien à cette quête découlant d'une crise, car elle « déplace l'attention vers le processus de création, rend la pensée libre, plus ouverte à ses contradictions, et communique au lecteur le mouvement de la réflexion autant que son résultat» (Lejeune et Bogaert, 2003, p. 10). Farhoud propose ainsi d'ouvrir une autoanalyse individuelle sur un ensemble élargi de subjectivités. Outre son dédoublement ( [1] e chemin de moi à moi »), la voix narrative multiplie les perspectives qui fluctuent entre « je » et « elle » ${ }^{38}$, auxquelles se greffent des «tu» (p. 76 et 89), des « vous» (p. 126 et 153) et

\footnotetext{
${ }^{37}$ N. se prépare à présenter des extraits de son récit axé sur un « voyage dans le Grand Nord» (p. 193); l'expression "perdre le nord » implique chez elle une désorientation et ses efforts pour restituer les points cardinaux de la navigation.

${ }^{38}$ Voir, à titre d'exemples, les chapitres 11,18 et 20.
} 
des «nous» (p. 63, 73, 85, 91 et 153), interpellant de la sorte des narrataires féminines, toutes cultures confondues :

Femme au singulier et au pluriel [...]. Femme africaine, orientale, extrême-orientale, américaine, latino-américaine, européenne et celle de l'Océanie [...], mariée, concubine, célibataire, en bonne santé ou grabataire, femme riche ou femme pauvre, Femme, Femme, Femme, [...] combien de deuils et des pertes dans une vie? (p. 12-13) ${ }^{39}$

C'est en fonction d'une telle hétérogénéité de sujets qu'émerge une dynamique d'interrogation et de contreinterrogation, sous-tendant "une narration en séquences » (Sniader Lanser, 1992, p. 256) ${ }^{40}$ ayant pour effet de suggérer l'encodage d'une multitude de voix féminines et l'évocation d'un faisceau d'expériences intimes relevant d'un socle commun de problèmes, que le féminisme, à son apogée, a eu tendance à minimiser (Ahmed, Kilby et al, 2000, p. 1-23). Qui plus est, on assiste à l'éclosion d'une (auto)représentation (darstellung) qui participe d'une déviation idiolectale par rapport à la forme classique du roman héroïque (Spivak, 2009, p. 51), grâce à laquelle $\mathrm{N}$. explore divers dilemmes corporels et psychiques propres à nombre de femmes subalternes.

S'inscrivant dans une configuration de privations qui caractérisent souvent l'approche de la maturité, ces dilemmes comprennent le retour d'âge, la rupture amoureuse et les deuils irrésolus qui constituent chez $\mathrm{N}$. autant d'écrans qui minent sa confiance et réfrènent son engagement dans des projets créateurs. De ces trois sources de dysphorie, elle parvient à

\footnotetext{
${ }^{39}$ Voici un autre exemple de ce genre d'apostrophe mêlant le singulier au collectif : "Pourquoi ai-je vécu tout ce temps... Pourquoi, Femme, pourquoi es-tu née, Femme, pourquoi vas-tu mourir? Qu'as-tu fait entre les deux? Pourquoi cette souffrance qui éclate dans ton ventre, Femme? » (p. 89)

${ }^{40}$ Nous traduisons ainsi les termes « sequential narration».
} 
gérer sa ménopause le plus facilement : «Mon corps est un pays en guerre. Avec francs-tireurs, couvre-feux, combats sanglants, cessez-le-feu, accalmies, reprises des négociations, reprises des combats. » (Farhoud, 2001, p. 99) Le sentiment qu'elle éprouve face au dépérissement de son corps est en partie régi par la nostalgie relative à une image conventionnelle de la jeunesse, celle dictée par les codes patriarcaux et capitalistes qui tendent à auréoler les femmes nubiles d'un surcroît d'estime et de désirabilité. Si N. contribue à lever le silence sur cette phase de la vie, souvent entourée d'essentialisme et d'âgisme ${ }^{41}$, elle la démystifie $^{42}$ surtout en montrant combien les tumultes corporels vécus par certaines femmes sont attribuables à la somatisation de problèmes psychiques, faisant ainsi référence à ses propres deuils repoussés. "Comment consommer le deuil, chaque deuil, tous les deuils? [...] Mais qu'est-ce que vivre quand nous n'avons plus personne, quand tête et corps se détraquent à tout moment sans prévenir [...] quand toutes les parties de notre corps nous font mal [...]?» (p. 73) ${ }^{43}$

Quant à la rupture amoureuse, N. éclaire non seulement sa propre malédiction, mais celle qui guette bien des femmes en dépit des modèles redéfinis du couple en vogue à son époque, abordant ainsi une autre facette contribuant à l'aliénation du sujet subalterne. Chez l'héroïne, la séparation provoquée par son compagnon s'avère si brutale qu'elle se sent complètement

\footnotetext{
${ }^{41}$ Citant Baudrillard, Sefovic (1996, p. 1) souligne que la ménopause demeure un signifiant sans signifié, car elle se définit en termes lacunaires (flétrissement corporel, dépendance sociale, conditions de vie débilitantes).

42 Dans Mrs. Dalloway, V. Woolf (1925) dénonce les stéréotypes associés à cette période de la vie.

${ }^{43}$ La perte est somatisée : « mon corps l'a emprisonnée dans mes reins, mon pancréas, mes lombaires » (Farhoud, 2001, p. 139-140) et N. doit l'évacuer, la rendre jusqu'à la conscience pour l'exhaler par des mots.
} 
désarçonnée, victime d'une "éventrure » (p. 22), d'une «perte de son identité » (p. 22). Elle se blâme ainsi d'avoir accepté de se plier à une position servile où elle a remplacé "être amoureuse par être dépossédée [...] et confondu [s]e donner et [s]e perdre » (p. 187) : « Est-ce que j'ai voulu le posséder au lieu de l'aimer? Le posséder en me dépossédant? » (p. 187) En vertu de sa dépendance affective envers un homme pour qui l'amour «n'est qu'une valeur parmi d'autres » (Beauvoir, 1949, p. 477), $\mathrm{N}$. incarne cette figure de l'amoureuse critiquée par Beauvoir; certaines femmes, écrit-elle, poussées par une recherche de transcendance, succombent à une «totale démission au profit d'un maître » (Beauvoir, 1949, p. 478), et demeurent ainsi « une narcissiste aliénée dans son moi » (1949, p. 486) :

[Une telle femme] éprouve aussi un désir passionné de déborder ses propres limites et de devenir infinie, grâce au truchement d'un autre qui accède à l'infinie réalité. Elle s'abandonne d'abord à l'amour pour se sauver; mais le paradoxe de l'amour idolâtre, c'est qu'afin de se sauver elle finit par se renier totalement. (Beauvoir, 1949, p.486; l'auteure souligne)

Cette définition encapsule le genre d'amour que N.a nourri pour son conjoint ${ }^{44}$, car son sentiment de ravissement s'accompagnait d'une admiration sans bornes de son talent, d'une impression de «bonheur béat » (Farhoud, 2001, p. 37) à l'écoute de sa «musique live» (p. 21; l'auteure souligne). Déplorant le cas de la femme de Mahler, qui, compositrice de formation, délaisse son travail créateur pour devenir simple «copiste» (p. 187), elle fustige celles qui acceptent d'être « cette esclave, cette servante, ce miroir trop docile, cet écho trop

44 «Il n'était pas seulement mon amour, il était mon jumeau, mon miroir.» (Farhoud, 2001, p. 21) 
fidèle » (Beauvoir, 1949, p. 503), soit un faire-valoir de la notoriété de l'autre :

Combien de femmes ont abandonné leur chemin pour [...] l'amour? Mais est-ce vraiment de l'amour? S'accomplir, est-ce la même chose pour une femme que pour un homme? [...] Derrière chaque homme, il y a une femme, dit-on, mais qui est là, derrière chaque femme, pour la pousser à s'accomplir? Pourquoi tant de femmes ont-elles glissé dans ce rôle? La société, oui, bien sûr, mais elles, chacune d'elles... et moi...? (Farhoud, 2001, p. 188)

L'héroïne a beau déplorer son égarement psychosocial et celui d'autres femmes ${ }^{45}$ dans l'espoir que celles-ci pourront tirer une leçon de son échec sentimental, sa blessure se ravive $\mathrm{au}$ moment où sa propre fille lui annonce son délaissement du métier musical dans le but de partir avec un jeune homme : «Le talent est une force vive, la musique, un engagement de chaque instant [...]; si ton premier engagement est l'amour d'un homme, tu placeras la musique au second plan. La musique ne supporte pas cela. Elle t'abandonnera!» (p. 26) Dans son autoanalyse, N. revient ainsi sur la nécessité de reconnaître le poids de l'idéologie patriarcale chez les femmes au plan de l'inconscient, et le besoin de travailler en fonction de son démantèlement (Spivak, 1987, p. 120).

Le départ de sa fille équivaut à un ultime arrachement, à la perte de «la dernière branche de [s]on arbre» (Farhoud, 2001, p. 25), cette béance la plongeant dans une quête existentielle qui traverse tout le roman (Simard [en ligne]). En vertu de cette filiation intergénérationnelle reposant sur

\footnotetext{
45 Des portraits de femmes animées d'un amour idolâtre sont élaborés par Annie Ernaux dans Passion simple (1991) et par Claire Martin dans « Le risque d'être dupe ", une nouvelle tirée de Toute une vie (1991).
} 
l'expression de soi, Splendide Solitude rejoint Le bonheur a la queue glissante, puisqu'à ses deuils s'ajoute celui relatif à sa propre mère, morte avec son conjoint dans un accident, ce qui la laisse orpheline à un jeune âge. Notons à cet égard que si l'initiation à la musique s'opère chez $\mathrm{N}$. sous une influence maternelle, sa mère étant une pianiste accomplie, c'est également à cette époque qu'elle choisit de s'en distinguer et de vivre par procuration (Marcheix, 2010, p. 172), d'être simple «témoin » (Farhoud, 2001, p. 39), une " caisse de résonance » (p. 38), par rapport à la musique d'autrui, si bien qu'elle saisit qu'« [elle] n'avai[t] pas ce besoin, ce désir qu'ont les artistes, cette urgence de s'exprimer qui les pousse à dépasser leurs peurs » (p.65). Le départ de sa fille en tant qu'ultime sevrage affectif se veut ainsi déclencheur d'une réflexion au sujet du talent qui va l'aiguillonner tout au long de son propre questionnement :

[Le] talent est une chose fragile, capricieuse, forte mais fragile, qui a besoin de tout notre égard pour se développer [...]. Le talent, c'est de la neige blanche qui descend du ciel, il faut être là pour en faire quelque chose, sinon la neige se salit ou bien se change en glace, en eau, elle s'évapore. Le talent [...] c'est un don que tu dois redonner. (p. 26)

Aux yeux de l'héroïne, le talent s'associe à la blancheur qui, du point de vue symbolique, est la couleur «de la révélation, de la grâce, de la transfiguration qui éblouit, éveillant l'entendement » (Chevalier et Gheerbrant, 1982, p. 146). Or à un moment stratégique où elle transcrit dans son cahier son objectif qui consiste à "recomposer [s]a vie» (Farhoud, 2001, p. 178), elle se voit mentalement entourée d'un tas de cendres : « [c]endre grise et légère et moi marchant dans cette cendre qui se soulève comme de la poussière grise chaque 
fois que je déplace un pied » (p. 178). Substance légère liée à l'obscurité, la cendre s'avère pesante symboliquement, car elle renvoie à un passé alourdi de son chagrin d'amour et de tous les deuils accumulés, entraves qui l'empêchent de se reconstruire. D'ailleurs, en se demandant « [c]omment nettoyer cette poussière grise, [...] [c]omment recommencer?» (p. 178) N. se rend compte qu'elle doit s'affranchir d'une identité sclérosée ainsi que de sa vulnérabilité affective avant de pouvoir gagner la confiance qui lui permettrait de passer à l'acte: risquer le déséquilibre, «tenir le crachoir», «me commettre » (p. 189) en "regardant ailleurs [...] dans une autre direction » (p. 178). Cela ne pourrait se faire qu'au moyen d'une conjuration de son passé et d'une initiation aux expériences d'extéroceptivité ${ }^{4}$, permettant de se réinvestir dans des activités créatrices propices au rapprochement de l'espace maternel refoulé.

Comment s'articule ce processus d'épuration psychique indissociable d'un travail de deuil? Quelle est sa fonction dans l'agentivité du sujet subalterne dès lors qu'il s'agit de se doter de compétences en vue d'une expression publique de la parole? À cet égard, N. ne pourra se défaire de son rôle de femme délaissée tant qu'elle n'aura pas fait le deuil de ses parents disparus. «C'est son départ à lui, lui, l'amour de ma vie, qui m’a

\footnotetext{
${ }^{46}$ Voir aussi : « Je me suis enfermée pour déterrer le passé [...] et l'enterrer d'une autre manière, avec un regard neuf qui changera peut-être même ce passé... Pour enfin faire de la place à ce qui va venir. » (p. 138)

47 Découlant du vocabulaire de la perception, la notion d'extéroceptivité renvoie au fait de percevoir par les sens ou l'esprit le monde extérieur et s'apparente respectivement à l'intéroceptivité, qui renvoie à la représentation du monde intérieur, et à la proprioceptivité, qui constitue l'interface entre les deux en tant que résonance sensible de l'expérience humaine. (Bertrand, 2000, p. 163)
} 
révélé la perte inacceptable de mes parents. Et le deuil à faire. » (p. 48) Une telle corrélation se défend dans la mesure où la perte de l'être aimé est décrite en termes de division survenue entre corps et esprit. Au moment de la rupture amoureuse, N. déclare : « Le choc a été trop grand. [...] J'ai fermé mon corps et mes sens. Je respirais tout juste.» (p.35) Une réaction similaire qualifie la crise vécue à la mort de ses parents, lorsqu'elle a ressenti « une dissociation entre [s]on corps et [elle]» (p. 184). Dans les deux cas, son attitude revient à ne pas "pardonner au destin » (p.190) de lui avoir infligé des coups aussi durs. S'appuyant sur les travaux de Mélanie Klein, Sylvie Metais (en ligne) explique que la capacité de surmonter les deuils auxquels est confronté chaque sujet sur le chemin de sa vie dépend de la façon dont les premiers deuils de l'enfance ont été vécus. Dans cette perspective, la notion de deuil englobe une signification plus large que celle désignant l'état causé par la mort d'un être aimé. Il est question du renoncement d'une image idéalisée de soi qui comble les besoins narcissiques chez l'être par la médiation des objets. Réfléchissant sur une pièce de théâtre portant sur le deuil, $\mathrm{N}$. arrive à cette même constatation, car elle admet que la perte de ce qu'on a aimé «n'a rien à voir avec la chose perdue, mais avec ce qu'elle représente pour nous - , la perte de ce qui nous compose, la perte de notre identité » (Farhoud, 2001, p.175). Dit autrement, pour se libérer de l'emprise subconsciente des êtres disparus, l'héroïne doit se détacher, en les transformant, des liens qui la ramènent à eux. Il s'agit non pas de perdre l'objet, mais de le retrouver autrement : «le travail de deuil est accompli lorsque le sujet parvient à sauvegarder l'amour pour l'objet et l'amour pour la vie » (Metais, en ligne). 
Pour y arriver, N. s'impose une période d' «entropie» (Farhoud, 2001, p. 188), réduit les distractions, s'enferme chez elle afin de réfléchir. Or ce travail de rumination s'accompagne de l'émergence d'un nouveau parcours perceptif (Fontanille, 1999, p. 9) qui va l'amener à assumer les pertes et à réorienter sa vision, rendant possible l'accès aux actes créateurs et à leur partage. Voilà pourquoi elle s'engage dans divers rites de purification visant à se délester du poids de son passé : l'assainissement du corps par une alimentation naturelle et de l'exercice physique, la marche dans son quartier et une randonnée à vélo jusqu'au Vieux-Montréal, l'élimination des fleurs séchées, le recouvrement des miroirs, la peinture du studio en blanc, ôtant ainsi les « empreintes grises » (Farhoud, 2001, p. 12) de l'ex-conjoint et le nettoyage du piano. Mais plus important, elle parvient à pleurer ses pertes, ce qui se produit lorsqu'en remontant une butte fréquentée à l'époque de sa vie familiale, elle «s'est assise, a regardé au loin et, pour la première fois depuis la mort de ses parents, ses larmes se sont mises à couler » (p. 163). Elle réussit ainsi à faire son deuil « en acceptant que la mort existe et qu'elle est là pour rester » (p. 140). L'expression d'une peine refoulée, conjuguée à la redirection du regard vers le lointain, représente un moment charnière de transformation. Non seulement elle accepte ainsi de «pardonner à la vie qui nous a fait si mal » (p. 175), mais consent à réorienter son regard vers l'avenir, au lieu de « [s]'acharner sur le passé » (p.181). Vivre enfin son deuil lui permet d'abolir la division du soi, ce qui se manifeste concrètement au niveau énonciatif dans les derniers chapitres par l'omniprésence d'une narration autodiégétique ${ }^{48}$.

\footnotetext{
${ }^{48}$ A partir du chapitre 53.
} 
Éléments d'une catharsis personnelle, les larmes endeuillées chez $\mathrm{N}$. sont en même temps une incitation à reconduire ses énergies dans le sens de la création :

Pleurez, Femme. Traversez la mer [...] les grandes eaux, dit le $Y i$ king, les épreuves, le difficile. Sachez que rien ne soulage tout à fait. Tout est à recommencer, pleurs et rires [...]. Splendide solitude. Les $S$ se transformeront en clés de sol, si tu le veux, Femme. Juste à tracer une ligne oblique et quelques frissons. (p. 153; l'auteure souligne)

Souligné par les psychanalystes, de Freud à Kristeva, ce travail de deuil va souvent de pair avec l'actualisation d'activités artistiques (Robin, 2003, p. 9-10), suggérée ci-dessus par une invitation à imaginer l'osmose des premières lettres éponymes (dont l'allitération frappe l'oreille) renvoyant à son périple existentiel en clés de sol. On observe se produire ce genre de métamorphose chez $\mathrm{N}$. En plus de recommencer à écrire quotidiennement ${ }^{49}$, elle s'inspire d'œuvres d'écrivains, de poètes, d'artistes et de cinéastes, et manifeste un attachement tout particulier pour Marceline DesbordesValmore (Farhoud, 2001, p. 106), dont le parcours de vie éprouvant, en tant que femme poète et comédienne, vivant de sa plume au début du $19^{\mathrm{e}}$ siècle, lui sert de modèle ${ }^{50}$. Les moments-clés de son expérimentation vocale arrivent lorsqu'elle s'essaie à la récitation d'un poème de Prévert accompagnée d'accords joués au piano, « en scandant chaque phrase, en laissant vibrer les sons et les mots » (p. 82) ${ }^{51}$, ce qui

\footnotetext{
${ }^{49}$ Sa pratique à tenir un journal remonte au départ de Zoubeida, une amie d'enfance perdue de vue (p. 159).

${ }^{50}$ Voir sa biographie : <http://www.desbordes-valmore.net/bio.html >.

${ }^{51}$ Il s'agit du poème intitulé « Le désespoir est assis sur un banc » (p. 82).
} 
est suivi par la récitation d'un poème de Lorca (p. 144) ${ }^{52}$, où une transcription de sonorités musicales la soulage dans un moment de détresse intense. Afin de se consoler, elle répète des maximes (p.131) et se chante des chansons (p. 153). Faisant partie de l'isotopie musicale qui sous-tend le roman, tous ces actes illocutoires réalisés en vue d'accéder à une compétence vocale d'artiste, traduisant au niveau de l'écrit, « un corps qui est dans l'épanchement, le dégorgement » (Cixous, 1976, p. 15), l'aident à sortir de son marasme. Ils témoignent du réveil de son âme poétique, dont elle s'était départie dans son obnubilation bourgeoise.

Du point de vue de l'affranchissement, N. se place sur la voie qui lui permettra de "trouver une raison de vivre" (Farhoud, 2001, p. 141). On assiste, chez le sujet subalterne, à l'acceptation de son vécu, à la célébration des métamorphoses du corps/esprit féminin (Gabilondo, 2003, p. 89). Qui plus est, les actes qu'elle accomplit relèvent d'une transformation de son champ de vision, à savoir l'adoption d'un nouveau regard extéroceptif, tant horizontal que vertical, cette modalité de l'être étant inspirée par une exposition prisée de Vallorz intitulée Cieli, consacrée à la représentation des nuances changeantes du ciel (Farhoud, 2001, p. 180). En modifiant le point focal de sa vision, ce qui l'amène à « [r]egarder le ciel au lieu de toujours regarder les briques » (ibid.), N. expérimente avec un nombre illimité de possibilités de capter la luminosité. Ce nouveau point de mire préfigure son réinvestissement dans ses propres aptitudes énonciatives, destinées à se matérialiser sur des «feuille[s] blanche[s]» (p.181), rappelant à la fois la

\footnotetext{
52 La lecture de l'« Élégie pour Jeanne la Folle » (p. 144) présuppose une voix intérieure déclamant chaque strophe.
} 
couleur du talent et celle de son studio qu'elle désire réintégrer (p. 196).

L'invitation lancée à ses amies de venir l'entendre offrir une description de « la beauté des paysages et des centaines de tons et textures du blanc à l'infini ... » (p. 194) observées durant le circuit tout intérieur de sa guérison, signale sa réhabilitation en tant que sujet agissant. $N$. exprime de cette manière son souhait d'évoluer à même une blancheur régénératrice, «symbole de la conscience diurne épanouie, qui mord sur la réalité » (Chevalier et Gheerbrabt, 1982, p. 146), de cultiver son propre chant, son talent d'interprète vocale. Affranchie de sa dépendance amoureuse et allégée des deuils du passé ${ }^{53}$, la protagoniste a finalement la confiance nécessaire pour briser son silence et se commettre : «[l]'intérieur n'existe que projeté à l'extérieur. L'intérieur n'existe qu'au moment où il prend forme. En paroles et en actes.» (Farhoud, 2001, p. 190) Émancipée par rapport à une idéologie de soumission domestique et forte d'une trajectoire ponctuée de reculs et d'avancées, par laquelle elle va "au bout de [s]oi-même » (p. 187), N. parvient à actualiser sa nouvelle identité à l'aide du corps et de la voix dans une sphère publique (Gabilondo, 2003, p. 88). Profitant de son gain de sagesse, l'héroïne sort définitivement de sa condition de subalterne et s'engage comme écrivaine et conteuse visant à être "entendue, respectée, crue, [et incarnant] un espoir d'influence » (Sniader

53 « Il faudra dorénavant que je me fabrique d'autres souvenirs [...]. Le présent, modifié par moi, deviendra peut-être le passé de mes jours à venir. » (p. 181) 
Lanser, 1992, p. 7) ${ }^{54}$. En somme, sa performance axée sur le «passage à l'autre » (Farhoud, 2001, p. 189) corrobore les propos d'Irigaray (1977, p. 67-82), qui maintient que, chez une femme, trouver une voix, c'est découvrir un chemin, une voie.

\section{De la brisure du mutisme à la réception d'une parole énoncée au féminin}

Pour clore cette exploration du sujet subalterne, soulignons que l'analyse a permis de révéler, chez nos protagonistes, la prégnance d'un phénomène que Rosi Braidotti nomme un processus de devenir-sujet: Dounia et N. sont, chacune à sa manière, propulsées par «[une] volonté de savoir, [un] désir de dire et [un] désir de parler» (Braidotti, 2014, p. 169) ${ }^{55}$. Explorant des dilemmes spécifiques ayant trait à l'éclatement de la conscience et de la voix chez des héroïnes situées à des moments précis d'une trajectoire de vie, Farhoud contribue à l'investigation d'une subjectivité féminine élargie, dépourvue de tout solipsisme mythique (Braidotti, 2014, p. 168), et favorise ainsi un échange continu d'approfondissements de la problématique avec d'autres créateurs. Peu importe leurs ancrages culturels et sociaux, les protagonistes féminins examinés, en vertu de leurs capacités idiolectales, décelées à l'aide d'un examen attentif des actes de langage adoptés,

\footnotetext{
54 Notre traduction : «writing a book is [...] a quest to be heard, respected, believed, a hope of influence ». Engagée dans l'élaboration d'un idiolecte personnel, $\mathrm{N}$. inscrit ainsi sa voix au niveau d'une doxa sociale.

55 Nous traduisons : "the entire process of becoming-subject is [a] will to know, a desire to say, [a] desire to speak ». D'après Braidotti (2014, p. 168), la subjectivité consiste en une rencontre d'instances réactives (potestas) et actives du pouvoir (potentia), abritées sous l'unité de la première personne du singulier « je ».
} 
participent d'une subjectivité féminine à la fois plurielle et affranchie que Braidotti qualifie de «subjectivité nomade» (p.163). D’après la critique, une telle notion de subjectivité infléchie par un modèle rhizomique de la pensée, propice aux communications d'ordre transversal, s'inscrit dans la révision des idéologies dominantes touchant au sujet. Cet effort de révision, qui rejoint le projet de Cixous, présuppose la compréhension d'une identité genrée, liée aux enjeux du savoir et du pouvoir, éléments permettant de nuancer toute forme de tendance vers un centrisme schématisant. Faire éclater la parole d'un sujet subalterne saisi dans sa diversité constitue de cette manière un trope iconoclaste, un lieu paratopique de résistance, allant à l'encontre de tout ordre de discours universalisant.

Un tel processus s'éclaire grâce à l'apport singulier de critiques littéraires à l'élargissement du décodage des stratégies signifiantes dont sont pourvus divers sujets subalternes (Gabilondo, 2003; Khannous, 2000; Vieira, 1995; Busia, 1989). Rappelons à cet égard que, dans sa démarche, Spivak se sert d'exemples de mutisme situés dans le cadre précis d'archives et d'études historiographiques appartenant aux contextes géopolitiques touchés par l'impérialisme, le (post)colonialisme et le patriarcat. Les concepts de représentation dont elle s'inspire s'inscrivent dans la problématique de la rature des sujets féminins des folios institutionnels officiels, où leur encodage ne dépasse pas souvent les quelques lignes d'une notice nécrologique (Spivak, 1999, p. 308). Reste que les outils herméneutiques avancés par Spivak, façonnés à partir de la dyade vertreten et darstellen, permettent d'identifier divers mécanismes qui contribuent à obscurcir la saisie de la voix de l'autre. Eu égard au domaine littéraire, nous avons vu que la représentation résulte toujours d'un travail engageant l'ethos et 
la praxis énonciative; elle est le produit d'une médiation où le réel se trouve reconfiguré par les enjeux de l'imaginaire et par un processus de symbolisation inhérent au langage poétique. Nous avons observé la rentabilité de ce volet inventif pour la mise au jour d'une parole souvent inaudible ou étouffée en considérant les parcours figuratifs et modaux chez nos héroïnes : dotée d'une voix monologique, Dounia redéploie à son avantage les astuces de l'oralité et l'usage du proverbe, propres à sa culture moyenorientale, pour conjurer un ensemble de souvenirs douloureux avant de se retirer de la vie. Dans Splendide Solitude, N. entreprend son autoanalyse en vertu d'un dédoublement et d'un dialogisme entamé avec la voix d'autres instances féminines (appartenant aux communautés élargies) et cette résonance vocale s'enrichit d'une intertextualité basée sur le langage des poètes, éléments qui la soutiennent dans son travail de deuil, dont dépend sa réalisation en tant que sujet artistique capable de livrer ses paroles publiquement. Dans sa démarche esthétique, Farhoud soumet la représentation littéraire aux modélisations et aux reconfigurations, montrant ainsi combien celle-ci a le potentiel de constituer un lieu puissant de résistance, postulat entièrement endossé par Cixous et par Braidotti : pour clamer sa vérité, le sujet subalterne adopte des moyens qui outrepassent les conventions d'une prise de parole standard (roman réaliste, encyclopédies, traités savants), notamment sous la forme de l'inscription à même la diégèse d'actes d'ordre communicatif allant du monologue écrit et du journal intime à l'énonciation de fragments discursifs hétérogènes et emboîtés, comme cela s'observe chez nombre d'écrivaines ${ }^{56}$. La prise en compte d'un

\footnotetext{
${ }^{56}$ Voir Leïla Sebbar, Les Carnets de Shérazade (1987), Ying Chen, Les Lettres chinoises (1993), Mariama Bâ, Une si longue lettre (2001) et Alice Walker, La Couleur pourpre ([1982] 2008); ces auteures intègrent au récit des journaux
} 
large diapason d'éléments porteurs de sens (relevant autant du signifié que du signifiant) participant de l'expression de soi permet en effet de saisir l'efficacité des scénographies transgressives pour contrer le mutisme du personnage féminin dans des textes littéraires qui traitent de sa socialisation (post)coloniale ou patriarcale (Busia, 1989).

Enfin, pour rompre le silence et s'exprimer, les personnages féminins marginalisés doivent rejoindre un réseau d'interlocuteurs. Spivak insiste sur ce critère de dissémination, soulignant que, même dans le cas d'un langage limite (1999, p. 308), afin d'être efficace, la parole du sujet subalterne doit sortir de l'intransitivité et rejoindre un(e) destinataire (Spivak, citée dans Landry et MacLean, 1996, p. 292) ${ }^{57}$. On observe sous cet angle l'aspect novateur de l'art romanesque farhoudien, car les enjeux de la réception font partie intégrante de l'intrigue de chaque roman analysé. Dounia offre son récit à Myriam, une écrivaine à succès, qui non seulement l'écoute, mais assiste sa mère dans l'élaboration énonciative de ses anecdotes et le retranscrit en langue française (à l'aide de transpositions et d'adaptations) pour en faire un livre destiné à rejoindre le grand public $^{58}$. Chez N., il faut croire que son dédoublement narratif ainsi que la lecture publique de son récit de voyage intérieur devant un ensemble de destinataires, susceptibles d'être émues par le timbre de la voix et l'exécution musicale qui l'accompagne, constituent les germes d'une transmission publique ayant le

intimes et des échanges épistolaires pour offrir aux personnages féminins un accès à la parole.

${ }^{57}$ Évoquant l'exemple d'une parole intransitive (voir la note 31), Spivak précise que, cinquante ans après son suicide, B. Bhaduri n'a pas pu communiquer son message aux descendantes de sa famille (1999, p. 308).

${ }^{58}$ Une telle transmission n'est jamais mimétique ni dépourvue de polémiques (Farhoud, 2004, p. 121). 
potentiel d'atteindre un auditoire plus vaste. La régularité d'une telle configuration, fondée sur une donation-partage de voix, où les interlocutrices se situent côté-à-côte et non en contreplongée, ou en position de domination l'une par rapport à l'autre, atténue le risque de chosification représentative (Weber-Fève, 2010, p.14-15) qu'appréhende Spivak. Forts de ce genre de dissémination de la vocalité intime à l'aide d'une réception figurée, les romans farhoudiens s'avèrent porteurs d'un discours éthique où les critères de médiation, de négociation et de fluidité vocales sont au principe de la mise en mots du «non-dit des vaincus, des femmes surtout » (Lequin, 2004). Entrant ainsi dans une circulation de signes artistiques qui dépasse les limites de la conscience solitaire, cette expression de soi, conjuguée à l'autre, est en mesure d'intégrer la doxa sociale en tant que manifestation idiolectale de l'identité, si bien qu'en suivant les analyses de Cixous et de Braidotti, elle pourrait s'intégrer dans des réseaux d'interférences élargis et multiples, permettant d'enrichir notre entendement de représentations fictives de l'altérité. En somme, le processus dialogique sous-tendant la mise au jour de voix féminines chez Farhoud permet au sujet subalterne de divulguer son histoire et d'inscrire sa parole dans les « circuits de la citoyenneté » (Spivak, 1999, p. 310) ${ }^{59}$, ce qui confirme ultimement son affranchissement par rapport à une position de mutisme.

\footnotetext{
${ }^{59}$ Il s'agit de notre traduction d'un extrait de l'énoncé suivant : « When a line of communication is established between a member of a subaltern group and the circuits of citizenship or institutionality, the subaltern has been inserted into the long road of hegemony. Unless we want to be romantic pursuits or primitivists about "preserving" subalternity - a contradiction in terms - this is absolutely desired. » (Spivak, 1999, p. 310)
} 


\section{Bibliographie}

AHMED, Sarah, Jane KILBY et al. (2000), Transformations. Thinking Through Feminism, Londres et New York, Routledge.

ANZIEU, Didier. (1995), Le moi-peau, Paris, Dunod.

ASHCROFT, Bill, Gareth GRIFFITHS et Helen TIFFIN. (2007), Post-Colonial Studies. The Key Concepts, Londres et New York, Routledge.

BÂ, Mariama. (2001), Une si longue lettre, La Madeleine-deNonancourt, Le Serpent à plumes.

BACCHILEGA, Cristina. (1995), "Writing” and "Voice": The Articulations of Gender in Folklore and Literature ", dans C. L. Preston (ed.), Folklore, Literature and Cultural Theory: Collected Essays, New York, Garland, p. 83-101.

BAKHTIN, Mikhail. (1981), "Discourse in the Novel», dans Michael Holquist (ed.), The Dialogic Imagination, Austin, University of Texas Press, p. 269-422.

BARTHES, Roland. (1982), L'Obvie et l'obtus. Essais critiques III, Paris, Seuil.

BENVENISTE, Émile. (1966), Problèmes de linguistique générale, t. I, Paris, Gallimard.

BERTRAND, Denis. (2000), Précis de sémiotique littéraire, Paris, Nathan.

BHABHA, Homi. (2007, [1994]), Les Lieux de la culture, Paris, Payot.

BOIREAU, Nicole. (1994), «Le monologue dans le théâtre féministe en Angleterre », dans Nicole Vigouroux-Frey (dir.), Voix de femmes à la scène, à l'écran, Paris, Presses universitaires de Rennes, p. 161-169. 
BOURDIEU, Pierre. (2000), Esquisse d'une théorie de la pratique précédé [sic] de trois études d'ethnologie kabyle, Paris, Seuil.

BOUSTANI, Carmen. (2003), Effets du féminin. Variations narratives francophones, Paris, Karthala.

BRAIDOTTI, Rosi. (2014), «Writing as a Nomadic Subject», Comparative Critical Studies, vol. 11, nos 2-3, p. 163-184.

BRYSON, Norman. (1986), Vision and Painting: the Logic of the Gaze, New Haven, Yale University Press.

BUSIA, Abena. (1989), «Silencing Sycorax: On African Colonial Discourse and the Unvoiced Female », Cultural Critique, no 14 , p. 81-104.

CANNONE, Belinda. (2001), Narrations de la vie intérieure, Paris, PUF.

CARRIÈRE, Marie et Catherine KHORDOC. (2006), «Deuils au pluriel. Sur deux textes d'Abla Fahroud », Voix et images, vol. 31, no 3 (93), p. 105-125.

CAVARERO, Adriana. (2005), For More Than One Voice: Toward a Philosophy of Vocal Expression, Stanford, Stanford University Press.

CHEN, Ying. (1993), Les Lettres chinoises, Paris, Babel.

CHEVALIER, Jean et Alain GHEERBRANT. (1982), Dictionnaire des symboles, Paris, Robert Laffont.

CHOUINARD, Marie-Andrée. (1998), «Ce que l'œil n’a pas vu, l'intelligence peut l'imaginer. La voix de Dounia », Le Devoir, 28 mars, p. D1.

CIXOUS, Hélène. (1976), "Le sexe ou la tête? », Les Cahiers du GRIF, no 13, p. 5-15.

COHN, Dorrit. (1981), La Transparence intérieure, Paris, Seuil. 
DAGHER, Joseph. (1994), «Parémiologie et village libanais : étude sociolinguistique de quelques matériaux », Arabica, t. 41 , p. $1-29$.

DE BEAUVOIR, Simone. (1949), Le Deuxième Sexe, vol. 2, Paris, Gallimard.

DESBORDES-VALMORE, Marceline. <http://www.desbordesvalmore.net/bio.html>.

ELIADE, Mircea. (1964), Traité d'histoire des religions, Paris, Payot.

EMETO-AGBASIÈRE, Julie. (1986), « Le proverbe dans le roman africain », Présence francophone, nำ 29, p. 27-41.

ERIKSON, Erik H. (1997), The Life Cycle Completed, New York, W.W. Norton.

ERNAUX, Annie. (1991), Passion simple, Paris, Gallimard.

FARHOUD, Abla. (1993), Les Filles du 5-10-15ф, Carnières, Lansman.

—. (1994), Quand j'étais grande, Solignac, Le bruit des autres.

—. (1997a), Jeux de patience, Montréal, VLB éditeur.

- . (1997b), Quand le vautour danse, Carnières, Lansman.

—. (1999), Maudite machine, Trois-Pistoles, Éditions TroisPistoles.

-. (2000), «Immigrant un jour, immigrant toujours ou comment décoller une étiquette ou se décoller de l'étiquette ", dans Anne de Vaucher Gravili (dir.), D'autres rêves. Les écritures migrantes au Québec, Venise, Supernova, p. 45-58.

—. (2003), Les Rues de l'alligator, Montréal, VLB éditeur.

—. (2001), Splendide Solitude, Montréal, Éditions de l'Hexagone. 
-. (2004 [1998]), Le bonheur a la queue glissante, Montréal, Éditions Typo.

- (2015), Toutes celles que j'étais, Montréal, VLB éditeur.

FEGHALI, Michel. (1938), Proverbes et dictons syro-libanais, Paris, Institut d'ethnologie.

FONTANILLE, Jacques. (1999), Sémiotique et littérature, Paris, PUF.

GABILONDO, Joseba. (2003), « The Subaltern Cannot Speak But Performs. Women's Public and Literary Cultures in Nineteenth Century Spain », Hispanic Research Journal, 5/1, p. 73-95.

GRAMSCI, Antonio. (1980), «Notes sur la question méridionale» (1926), dans Écrits politiques III - (19231926), Paris, Gallimard.

GREIMAS, Algirdas. (1970), Du sens. Essais sémiotiques, Paris, Seuil.

HAYDAR, Adnan. (1989), « The Development of Lebanese Zajal: Genre, Meter, and Verbal Duel», Oral Tradition, 4/1-2, p. 189-212.

HUBIER, Sébastien. (2003), Littératures intimes, Paris, Armand Colin.

IRIGARAY, Luce. (1977), Ce sexe qui n'en est pas un, Paris, Minuit.

JARDINE, Alice. (1991), Gynésis. Configurations de la femme et de la modernité, traduit par Patricia Baudoin, Paris, PUF.

JOHNSON, Erika L. et Patricia MORA. (2013), The Female Face of Shame, Bloomington, Indiana University Press.

KHANNOUS, Touria. (2000), « The Subaltern Speaks: Remaking /Her/Story/ In Assia Djebar's La Nouba des femmes au Mont Chenoua », dans Maureen Eke, Kenneth W. Harrow et 
Emmanuel Yavak (dir.), Africain Images: Recent Studies and Text in Cinema, Trenton, African World Press, p. 51-71.

KRISTEVA, Julia. (1969), Sèméiotikè. Recherches pour une sémanalyse, Paris, Seuil, coll. « Tel quel ».

LANDRY, Donna et Gerald MACLEAN (dir.). (1996), The Spivak Reader, New York and Londres, Routledge.

LAURENCE, Margaret. (2012 [1964]), L'Ange de pierre, traduit par Sophie Bastide-Foltz, Montréal, Éditions Alto.

LEJEUNE, Philippe et Catherine BOGAERT. (2003), Histoire d'une pratique. Un journal à soi, Paris, Les Éditions Textuels.

LEQUIN, Lucie. (2004), "Abla Farhoud et la fragilité du bonheur», Rocky Mountain E-Review of Language and Literature, vol. 58, $\mathrm{n}$ - 1 , printemps, <http://rmmla.innoved.org/ereview/58.1/articles/lequin.asp>.

MACHEREY, Pierre. (1966), Pour une théorie de la production littéraire, Paris, Maspero.

MAILLET, Antonine. (1990 [1971]), La Sagouine, Montréal, Bibliothèque Québécoise.

MARCHEIX, Daniel. (2010), Les Incertitudes de la présence, Berne et Bruxelles, Peter Lang.

MARTIN, Claire. (1999), "Le risque d'être dupe », dans Toute la vie, Montréal, L'instant même, p. 69-74.

MARX, Karl. (1984 [1851]), Le Dix-Huit Brumaire de LouisNapoléon Bonaparte, trad. rev. par G. Cornillet, Paris, Messidor/Les Éditions sociales.

MAINGUENEAU, Dominique. (2004), Le discours littéraire. Paratopie et scène d'énonciation, Paris, Armand Colin.

-. (1993), Le Contexte de l'ouvre littéraire. Énonciation, écrivain, société, Paris, Dunod. 
MESCHONNIC, Henri. (1976), «Les proverbes, actes de discours », Revue des sciences humaines, no 163, p. 419-430.

METAIS, Sylvie. « Deuil », dans Encyclopaedia Universalis, <http : //www.universalis-edu.com/encyclopedie/deuil/>.

MIMOUNI, Isabelle et Lucie ROBERT. (2002), « Théories de la narration », dans P. Aron, D. Saint-Jacques et A Viala (dir.), Le Dictionnaire du littéraire, Paris, PUF, p. 597-598.

MONTANDON, Alain. (2006), «Abla Farhoud: portrait d'une libanaise en exil », Neohelicon, 33, 1.7, p. 81-90.

MORTON, Stephen. (2011), «Subalternity and Aesthetic Education in the Thought of Gyatari Chakravorty Spivak ", Parallax, vol. 17, no 3, p. 70-83.

MULVEY, Laura. (1975), "Visual Pleasure and Narrative Cinema », Screen, vol. 16, no 3, automne, p. 6-18.

OORE, Irène. (2000), « Le mutisme dans Le bonheur a la queue glissante d'Abla Farhoud (1998) et dans Un sourire blindé de Sergio Kokis (1998) », dans Robert Viau (dir.), La Création littéraire dans le contexte de l'exiguïté, Beauport, Publications MNH, p. 473-483.

PROULX, Patrice J. (2004), "Migration and Memory in MarieCélie Agnant's La Dot de Sara and Abla Farhoud's Le bonheur a la queue glissante », dans Susan Ireland et Patrice Proulx (dir.), Textualizing the Immigrant Experience in Contemporary Quebec, Westport, Praeger Publishers, p. 124-136.

RABATÉ, Dominique. (1999), Poétiques de la voix, Paris, José Corti.

ROBIN, Régine. (2003), Le Deuil de l'origine, Paris, Kimé.

SAID, Edward W. (1994), Culture and Imperialism, New York, Alfred A. Knopf Inc.

SCHLINK, Bernhard. (1996), Le Liseur, Paris, Gallimard. 
SEARLE, John R. (1972), Les Actes de langage, Paris, Hermann.

SEBBAR, Leïla. (1987), Les Carnets de Shérazade, Paris, Stock.

SEFCOVIC, Eve. (1996), "Stuck in the Middle: Representations of Middle-Aged Women in Three Popular Books About Menopause ", Women's Studies in Communication, vol.19, no 1, p. 1-27.

SIMARD, Hélène, «Abla Farhoud : la solitude est un cercueil de verre », Entrevues littéraires,

$<$ http://revue.leslibraires.ca/entrevues/literaturequebecoise/abla-farhoud $>$.

SNIADER LANSER, Susan. (1992), Fictions of Authority. Women Writers and Narrative Voice, Ithaca and Londres, Cornell University Press.

SORIANO, Marc. "Proverbes », dans Encyclopaedia Universalis, $<$ http://www.universalis-edu.com/encyclopedie/proverbes/>.

SPIVAK, Gayatri Chakravorty. (1988), "Can the Subaltern Speak?", Marxism and the Interpretation of Culture, dasn L. Grossberg et C. Nelson (dir.), Urbana and Chicago, University of Illinois Press, p. 271-313.

-. (1988), In Other Worlds. Essays in Cultural Politics, New York, Routledge.

-. (1999), A Critique of Postcolonial Reason, Cambridge, Harvard University Press.

-. (2009), Les subalternes peuvent-elles parler?, traduit par Jérôme Vidal, Paris, Éditions Amsterdam.

STANDFORD FRIEDMAN, Susan. (1996), «Beyond Gynocriticism and Gynesis: The Geographies of Identity and the Future of feminist Criticism», Tulsa Studies in Women's Literature, vol. 15, no 1, printemps, p. 13-40.

STEINEM, Gloria. (1992), Revolution from Within, Toronto / London, Little, Brown and Co. 
VERDUYN, Christl. (2001), "Femme(s) sans lettres », dans Lucie Lequin et Catherine Mavrikakis (dir.), La Francophonie sans frontière, Paris, L'Harmattan, p. 295-306.

VIEIRA, Else. (1995), "Can Another Subaltern Speak/Write? », Renaissance and Modern Studies, no 38, p. 96-125.

WALKER, Alice. ([1982] 2008), La Couleur pourpre, traduit par Mimi Perrin, Paris, Robert Lafont.

WEBER-FÈVE, Stacey. (2010), Re-hybridizing Transnational Domesticity and Femininity. Women's Contemporary Filmmaking and Lifewriting in France, Algeria and Tunisia, Lanham, Boulder, New York, Toronto, Plymouth, Lexington Books.

WOOLF, Virginia. (1970 [1942]), "Professions for Women», dans The Death of the Moth and Other Essays, New York/Londres, Harcourt, Brace, Jovanovich, p. 235-242.

—. (1925), Mrs. Dalloway, Londres, Hogarth Press.

\section{Résumé}

Nous examinons les configurations du sujet subalterne dans deux romans d'Abla Farhoud à partir d'un cadre théorique inspiré des postulats élaborés par Gayatri Chakravorty Spivak relatifs à la rature représentative de l'archive dont sont victimes les femmes subalternes et aux défis d'ordre herméneutique associés à la saisie de leur "voix-conscience». Les enseignements de Spivak sont enrichis par une prise en compte de la réflexion d'Hélène Cixous ayant trait à l'aphonie féminine et à l'inscription d'une parole empreinte d'altérité à l'intérieur de la circulation des signes. Nous penchant sur les cas de Dounia dans Le bonheur a la queue glissante (2004 [1998]) et de la narratrice de Splendide Solitude (2001), nous cherchons à éclairer le mutisme féminin et à cibler les 
modalités énonciatives et rhétoriques non-canoniques adoptées par Farhoud pour rendre audible une voix habituellement réprimée. L'examen des enjeux de la vocalité (Maingueneau) chez ces personnages engagés dans un processus du devenirsujet (Braidotti), relevant d'une trajectoire féminine saisie à rebours, permet d'enrichir notre compréhension de la poétique représentative et de l'axiologie éthique chez Farhoud.

\section{Abstract}

Consisting of an examination of the subaltern subject in two of Abla Farhoud's novels, the following study adopts a theoretical framework based on Gayatri Chakravorty Spivak's investigations into voice silencing with respect to marginal female characters affected by imperialistic or patriarchal mechanisms and the hermeneutics of retrieval of female conscience. Spivak's teachings are enriched by concepts developed by Hélène Cixous on the absence of female voice within logocentric hegemony and on subversive strategies by which the female voice is inscribed within universal sign systems. In scrutinizing the cases of Dounia in Le bonheur a la queue glissante (2004 [1998]) and the female narrator in Splendide Solitude (2001), the article elucidates the problem of mutism and offers an analysis of non-canonical enunciative and rhetorical attributes devised by Farhoud in order to express a thwarted female voice. Examining aspects of vocalité (Maingueneau) in female characters involved in a process of becoming (Braidotti), during two periods of a feminine trajectory presented in a reversed order, the study enriches our understanding of Farouhd's representative poetics and axiological ethics. 